\title{
Systematic review and meta-analysis of the effect of protein and amino acid supplements in older adults with acute or chronic conditions
}

\author{
Heilok Cheng ${ }^{1}$, Jielan Kong ${ }^{1}$, Catherine Underwood $^{1}$, Peter Petocz ${ }^{2}$, Vasant Hirani ${ }^{1}$, Beryl Dawson ${ }^{3}$ \\ and Fiona O'Leary ${ }^{1 *}$ \\ ${ }^{1}$ Nutrition and Dietetics Group, School of Life and Environmental Science, Charles Perkins Centre, University of Sydney, \\ Camperdown, NSW 2006, Australia \\ ${ }^{2}$ Department of Statistics, Macquarie University, North Ryde, NSW 2109, Australia \\ ${ }^{3}$ Nutrition and Dietetics, Balmain Hospital, Sydney Local Health District, Balmain, NSW 2041, Australia
}

(Submitted 29 December 2016 - Final revision received 18 November 2017 - Accepted 12 December 2017)

\section{Abstract}

The loss of lean body mass, muscle strength and physical function causes significant problems in older adults. Protein and amino acid supplements can preserve muscle strength but the effect on function is variable. We conducted a systematic literature review and metaanalysis to investigate the effect of protein and amino acid supplementation on fat-free mass, muscle strength and physical function in malnourished, frail, sarcopenic, dependent or elderly with acute or chronic conditions, with or without rehabilitation exercise. Databases searched included Medline, BIOSIS, CINAHL, Cochrane Library, EBM Reviews, Embase, Pre-Medline, ProQuest, PubMed and Scopus. Retrieved articles were assessed by two reviewers using the Cochrane Risk of Bias (ROB) Tool. In all, thirty nine randomised controlled trails ( $n$ 4274) were included. The studies used a range of protein or essential amino acid (EAA) supplements in a variety of settings, including hospital, community and long-term care. Only seven studies had low ROB and no effect of supplementation was found on any outcomes. Analysis of all thirty-nine studies suggest protein and EAA supplements may improve fat-free mass, muscle strength and physical function (standardised mean difference $0 \cdot 21-0 \cdot 27$, all $P<0 \cdot 005$ ), but significant heterogeneity and ROB was evident. Predetermined subgroup analysis found undernourished elderly benefitted most; EAA were the most effective supplements and small beneficial effects were seen without rehabilitation exercise. The high heterogeneity and few studies with low ROB limits the conclusions and more high quality studies are needed to determine the best nutritional strategies for the maintenance of strength and function with increasing age.

Key words: Dietary proteins: Sarcopenia: Physical function: Muscle strength

Advancing age is accompanied by progressive decline in skeletal muscle mass, leading to the development of sarcopenia ${ }^{(1)}$, which results in significant disability, mortality and health care costs $^{(2,3)}$. Sarcopenia affects $5-13 \%$ of $60-70$ year olds and up to $50 \%$ in those aged more than 80 years with higher prevalence in elderly with disease conditions ${ }^{(4)}$. The world's ageing population is expected to reach 1.5 billion in $2050^{(5)}$ and due to the increased use of health and aged care resources, it is important to determine ways to maintain and improve the health of elderly people before muscle deterioration worsens life outcomes.

Preservation of muscle mass and stimulation of muscle protein synthesis (MPS) through nutritional intervention is a potential therapy for sarcopenia ${ }^{(6,7)}$. MPS is stimulated by protein and amino acid (AA) intake ${ }^{(6,8-10)}$. In older adults, inadequate dietary intake of protein ${ }^{(7,10)}$ and the decreased anabolic response to dietary protein intake are believed to contribute to the loss of muscle mass ${ }^{(8,11)}$. Research has further indicated that essential amino acids (EAA) are key regulators of MPS, with leucine a potent muscle stimulator ${ }^{(12,13)}$ and may suppress muscle protein breakdown ${ }^{(10)}$. EAA supplements containing leucine have produced improvements in physical function in some studies ${ }^{(14-16)}$. Dosage and timing of protein intake may also contribute, with studies $^{(17,18)}$ demonstrating that protein consumed in $\geq 30 \mathrm{~g}$ bolus intakes are more effective in increasing post-prandial plasma AA concentration, a signal that stimulates MPS ${ }^{(18)}$, and may have the additive effect to overcome the anabolic threshold to MPS ${ }^{(17)}$.

Recent reviews have shown supplements, including high protein oral nutritional supplement $(\mathrm{ONS})^{(19)}, \mathrm{EAA}^{(20)}$ or higher protein diets ${ }^{(21)}$ improve muscle strength in older adults, but these reviews included healthy and frail elderly participants and included studies using resistance training $(\mathrm{RT})^{(19-22)}$. Although RT is effective in improving muscle strength, it may not be viable for all patients. Nutritional supplementation alone or in association with geriatric rehabilitation may be a practical

Abbreviations: AA, amino acid; EAA, essential amino acid; FFM, fat-free mass; MPS, muscle protein synthesis; ROB, Risk of Bias; RT, resistance training; SMD, standardised mean difference.

* Corresponding author: F. O’Leary, email fiona.oleary@sydney.edu.au 
method for improving muscle mass and function, particularly in vulnerable participants such as those that are hospitalised, malnourished or sarcopenic and who are unwilling or unable to undertake RT.

The aim of this meta-analysis, therefore, is to identify evidence for the effectiveness of protein supplements on muscle mass, strength and physical function in malnourished, frail, sarcopenic, dependent or elderly with acute or chronic conditions, alone or in association with rehabilitation, but not undertaking RT and mirrors usual care provided for the elderly.

\section{Methods}

This meta-analysis used the Preferred Reporting Items for Systematic Reviews and Meta-Analyses Guidelines ${ }^{(23)}$ (online Supplmentary Table S1). A literature search was conducted in April 2014 and updated on 17 June 2016, in the databases Medline, BIOSIS, CINAHL, Cochrane Library, EBM Reviews, Embase, Pre-Medline, ProQuest, PubMed and Scopus. Search terms used were synonyms and combinations of: dietary protein, whey, leucine, AA, nutritional status, nutrition supplement, skeletal muscle, muscle weakness, muscle atrophy, muscle strength, muscle function, sarcopenia, cachexia, weight changes, body composition, malnutrition, frailty, elderly and ageing. The search strategy appears in the online Supplementary Table S2. Citations were downloaded into Endnote X7. Interventions retrieved involved participants with mean age $\geq 65$ years and enrolled volunteers aged 60 years or older, and compared increasing protein intake through protein-rich sources of food; food fortification; and orally consumed liquid, powder or tablets containing protein and energy or EAA, as EAA mixtures or single EAA, for example, leucine to a placebo; supplement of lower protein and energy content; usual care; or no dietary intervention control group. Studies of dietary advice alone were not included. We included studies enrolling hospitalised, community-dwelling or institutionalised subjects with conditions such as frailty, malnutrition, sarcopenia, post-orthopaedic surgery, fracture or acute disease. Studies focusing solely on RT, enteral or parenteral nutrition, or with young, well or healthy adults, or older adults with cancer, cirrhosis, renal disease, stroke or gastrointestinal surgery were excluded. Studies with aerobic or physical rehabilitation activities, without use of RT (defined as $60 \%$ of one-repetition maximum) ${ }^{(24)}$, were included only if identical rehabilitation components were provided to both intervention and control groups.

Titles and abstracts were checked for relevance, and coded for retrieval or exclusion. Reference lists of included studies and systematic literature reviews were checked for additional articles. Retrieved articles were coded and assessed by two reviewers using the Cochrane Risk of Bias (ROB) Tool ${ }^{(25)}$. Study data from eligible studies were extracted and tabulated for participant characteristics, numbers and withdrawals; type, dose, frequency of intervention; compliance to intervention; and outcome measures for fat-free mass (FFM), muscle strength, and physical function. In order to ascertain the most effective interventions in well-designed studies, studies with low protein density ONS ( 10 to $<15 \%$ energy from protein) or high $\mathrm{ROB}$ were excluded from the analysis ${ }^{(26-39)}$.

\section{Data extraction}

Results for FFM, muscle strength, and physical function were extracted and analysed separately, either as changes from baseline, or baseline and endpoint scores. If the mean and standard deviation were not provided, the median was used and the SD estimated from the interquartile range. Sensitivity analyses indicated that leaving out one study at a time, did not change overall results appreciably. A correlation of 0.75 was used when variance data of the mean difference were not available $^{(40)}$. Sensitivity analyses was undertaken and showed that the exact correlation value used made little difference to the overall results. Where data was reported without a quantitative value, that is in graphs or figures, study authors were contacted for further information. More than one outcome measure was commonly provided for the outcome of physical function; however, only one measure was included. The outcome measure chosen for inclusion was based on the characteristics of the instrument, that is tools that measured rather than collected self-reported information, and tools testing more advanced functional outcomes, for example walking speed was selected over Katz Activities of Daily Living (ADL). Tools administered more frequently in studies were used in an effort to reduce variability; for example, tests of walking speed were assessed in seven studies ${ }^{(33,41-46)}$ and was the measure selected where data was available. If data for multiple time-points were available, the data closest to intervention end were used, as this was the most common timepoint analysed.

\section{Subgroup analysis}

Pre-determined subgroup analyses were performed based on ROB score, participant characteristics, protein intervention type, and if participants undertook aerobic or physical rehabilitation activities. Participant characteristics were grouped by trial setting (hospital, long-term care (LTC) in institutions, or community) and nutritional status (nourished or undernourished) as defined by study authors, or with low body weight or BMI $<25 \mathrm{~kg} / \mathrm{m}^{2}$ as used in a previous review ${ }^{(47)}$. Interventions were characterised as protein-rich foods; EAA (as EAA mixtures or single types of EAA); ONS with protein density of 10 to $<15 \%$ (low), 15 to $\leq 20 \%$ (moderate) or $>20 \%$ (high) composition of total energy; and whey-based ONS. Regression based on study duration was also undertaken.

Post hoc analyses was undertaken to determine potential effects of additional nutrients (intrinsically in protein-rich foods, or added as a supplement, e.g. in ONS) and differential effects in studies using a placebo, with meta-regression based on grams of protein or EAA provided by the intervention to determine the effect of dose.

\section{Statistical analysis}

Meta-analysis was performed using Comprehensive MetaAnalysis version 2 (Biostat) using the random effects model for heterogeneous samples, as participants were of various health statuses and heterogeneity was likely to be high. Heterogeneity was analysed using the $I^{2}$ statistic $^{(48)}$, with 
$P$ values used to identify statistically significant heterogeneity. No significant heterogeneity was found for the FFM analysis; however, the random effects model was used across the three outcome variables for consistency. Effect size was reported as the standardised mean difference (SMD) and the 95\% CI. General rules of thumb for effect size used were 0.2 for 'small', 0.5 for 'moderate' and 0.8 for 'large' effect ${ }^{(49)}$. Overall significance was assumed at $P<0.05$. Meta-regression on additional EAA and protein dose provided through interventions and intervention length were undertaken and presented as slope, standard error. Meta-analysis data were presented as forest plots, and publication bias was assessed with funnel plots.

\section{Results}

A total of 8702 citations were reviewed, with thirty-nine studies fulfilling the selection criteria (Fig. 1).
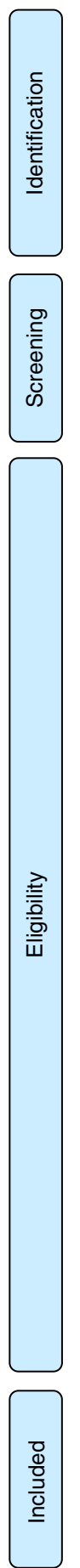

Records identified through database searching ( $n$ 6452)
Additional records identified through other sources ( $n$ 84)

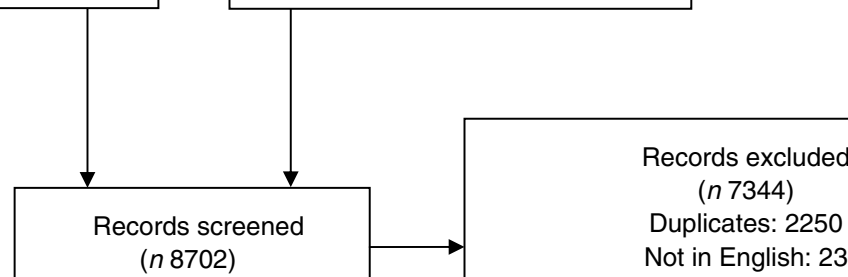

Not a relevant outcome: 11

Not a relevant study: 5060

Full-text articles excluded, with reasons ( $n$ 1298)

Could not be retrieved: 21

Not in English: 4

Not an RCT: 26

Not original research: 285

Not a relevant outcome: 105

Other types of supplements: 215

Not a relevant population: 263

No age group given for participants: 1

Not a relevant study: 147

Uses enteral or parenteral nutrition: 71

Unwell subjects with resistance training: 27

Well subjects with resistance training: 28

Well subjects with supplementation: 50

Unwell subjects with aerobic training

but not supplementation: 4

Well subjects with aerobic training: 2 Unwell subjects with supplementation but no relevant outcomes: 49

Records excluded (n21)

Unwell subjects with protein

Studies suitable for qualitative synthesis $(n 60)$

supplementation and relevant outcomes but not usable data: 7

Studies with high risk of bias or low-density protein supplements: 14

Fig. 1. Preferred Reporting Items for Systematic Reviews and Meta-Analyses flow diagram summarising selection process. RCT, randomised controlled trails. 


\section{Publication bias and sensitivity analysis}

No obvious evidence of publication bias was found for studies of FFM and physical function, with only minor evidence of publication bias for studies of muscle function, as indicated by the funnel plots (see online Supplementary Fig. S1(a)-(c)). Sensitivity analysis showed small changes in SMD after removal of one study in each outcome analysis, but $P$ values remained significant (all $P<0 \cdot 01$ ), and all thirty-nine studies were included in the analysis. The weights of individual studies ranged from 2.18 to 12.51 for FFM, 2.05 to 5.10 for muscle strength, and 1.02 to 5.48 for physical function.

\section{Characteristics of included studies}

A summary of the main characteristics of the thirty-nine studies that met inclusion criteria can be found in Table 1. Two authors who were contacted provided additional information not reported in the original studies ${ }^{(50,51)}$. The included studies involved 4274 participants, with ages ranging from $60^{(43,52)}$ to $103^{(53)}$. In all, sixteen studies involved community-dwelling participants ( $n$ 2350); twelve in hospital ( $n$ 1264); and eleven in LTC settings, including residential care and nursing homes ( $n$ 660). Most of the studies included undernourished, at risk of malnutrition, sarcopenic or frail participants, with thirteen studies including elderly participants with a mean normal weight:height ratio (BMI) $\geq 25 \mathrm{~kg} / \mathrm{m}^{2}$.

Thirty-one studies involved supplementation with ONS and protein-rich foods ${ }^{(41,43,44,46,50,51,53-77)}$, three of which used whey protein-based ONS ${ }^{(56,57,73)}$. Eight studies used EAA supplementation, either as EAA mixtures with or without other nutrients ${ }^{(14,16,45,52,78,79)}$ or leucine supplementation only ${ }^{(80)}$. Study duration ranged from 2 weeks ${ }^{(77)}$ to 24 months ${ }^{(62)}$, with twenty-six to 1006 participants included. The health characteristics of participants varied, with ten studies focusing on frail participants $(n 781)$; three studies on sarcopenic participants ( $n$ 488); and fifteen studies with participants who were malnourished, undernourished or at risk of malnutrition ( $n$ 2066). The remaining studies included participants diagnosed with, or recovering from infections or acute illness ( $n 550$ ); fractures or other surgical operations ( $n$ 540); wound healing difficulties ( $n$ 38); and catabolic diseases ( $n$ 30). Nine studies involved participants with long-term conditions, such as including coronary artery disease, chronic heart failure, type 2 diabetes mellitus, chronic obstructive pulmonary disease, osteoporosis, the metabolic syndrome and dementia ( $n$ 517).

Of the thirty-one studies with ONS and protein-rich food interventions, sixteen studies used attention control, no dietary intervention, or standard dietetic care as a comparator; eleven studies used a protein-free comparator; one used a protein-free placebo; and three used alternative ONS with lower energy and protein content. Interventions in these studies could increase energy and protein intake by $355 \mathrm{~kJ}(85 \mathrm{kcal})^{(71)}$ to $5040 \mathrm{~kJ}$ $(1200 \mathrm{kcal})^{(68)}$, and $4^{(71)}$ to $50 \mathrm{~g}^{(64)}$, respectively. Mean compliance for ONS intake varied from 54 to $100 \%$.

Of the eight studies using EAA supplementation, five studies used placebos as comparators, and the remaining studies used attention control, no dietary intervention or standard dietetic care. EAA and leucine intake could increase by $3^{(79)}$ to $40 \mathrm{~g} / \mathrm{d}^{(52)}$, and
$1 \cdot 2^{(79)}$ to $7.5 \mathrm{~g} / \mathrm{d}^{(80)}$, respectively. Only three studies reported compliance to supplements and comparators, of 72 to 100 and $72 \%$, respectively.

A total of twelve studies involved aerobic or physical rehabilitation activities, which included use of activities as part of standard care ${ }^{(52,59,69,75)}$; participants recruited from rehabilitation units ${ }^{(50)}$; participants discharged from hospital to rehabilitation facilities ${ }^{(67,68,72)}$; and as novel health interventions $^{(43,57,73,76)}$.

\section{Risk of bias assessment}

Seven studies were at low and thirty-two at unclear ROB. Intention to treat analysis was carried out in eleven studies. Randomisation was inadequately reported in nine stu$\operatorname{dies}^{(53,55,57,60,66,71,75-77)}$. Participants were blinded in only nineteen studies ${ }^{(14,16,43,44,46,52,56,58,61,63,65,67,70,72-74,78,80,81)}$, mainly due to lack of use of a placebo; researchers were blinded in eighteen studies $^{(14-16,41,45,46,52,54,56,61,63,69,72-74,78,80,81)}$ and outcome assessors in fifteen studies ${ }^{(41,43,45,46,51,56,59,61,62,67-70,74)}$.

\section{Outcomes}

Forest plots from the analysis of FFM, muscle strength and physical function outcomes can be found in Figs 2-4, respectively. Statistics, including heterogeneity for all subgroup analyses are reported in the online Supplementary Table S3 with significant effect size results reported below. Studies were alphabetically coded 'a' or 'b' for studies with multiple intervention arms ${ }^{(55,79)}$ or if outcome reporting was separated by intervention subgroups ${ }^{(75)}$.

\section{Body composition}

In all, sixteen studies reported data on FFM (analysed $n$ 970) with methods including dual-energy X-ray absorptiometry and bioelectrical impedance analysis. The effect of ONS and EAA supplementation on FFM was significant with a small effect size (SMD 0.21; 95\% CI 0.07, 0.35, P<0.01, no evidence of heterogeneity, $I^{2}=15 \cdot 1, P=0 \cdot 28$ ) (Fig. 2 ). Only one study had low $\mathrm{ROB}^{(74)}$, with no significant effect on FFM seen. However, a small effect was seen in studies with unclear ROB (SMD 0.23; $95 \%$ CI $0 \cdot 08,0 \cdot 37, P<0 \cdot 01$, no evidence of heterogeneity, $\left.I^{2}=17 \cdot 6, P=0 \cdot 25\right)$. Other subgroup analysis showed improvements in FFM in studies using protein-rich foods (SMD 0.26; $95 \%$ CI $0.02,0.50, P=0.03$, no evidence of heterogeneity, $I^{2}=0 \cdot 0, P=0 \cdot 43$ ); studies using supplements with additional vitamins or minerals (SMD 0.24; $95 \%$ CI 0.09, 0.38, $P<0 \cdot 01$, no evidence of heterogeneity, $\left.I^{2}=0 \cdot 0, P=0.56\right)$ and with patients not participating in rehabilitation programmes (SMD 0.23; $95 \%$ CI $0 \cdot 06,0 \cdot 41, P=0 \cdot 01$, no evidence of heterogeneity, $I^{2}=29 \cdot 3$, $P=0 \cdot 14$ ) (online Supplementary Table S3). Meta-regression of the protein (slope $0 \cdot 007$, sE $0 \cdot 012, P=0.57$ ) or EAA (slope $0 \cdot 08$, SE $0 \cdot 15, P=0 \cdot 60$ ) dose provided in interventions showed no evidence of dose-response, although the number of EAA studies was small ( $n$ 4). Duration of intervention also showed no significant effect (slope $-0 \cdot 003$, se $0 \cdot 007, P=0 \cdot 62$ ). Studies $(n 4)$ using a true placebo showed no significant effect. 


\section{N British Journal of Nutrition}

Table 1. Characteristics of included studies

\begin{tabular}{|c|c|c|c|c|c|c|c|c|}
\hline $\begin{array}{l}\text { First author, } \\
\text { year }\end{array}$ & $\begin{array}{l}\text { Study } \\
\text { ROB }\end{array}$ & $\begin{array}{l}\text { Population sample size } \\
\text { (IG/CG) }\end{array}$ & $\begin{array}{l}\text { Mean age (years), } \\
\text { baseline BMI }\left(\mathrm{kg} / \mathrm{m}^{2}\right)\end{array}$ & Study length & Ingestion details & $\begin{array}{l}\text { Dose. Total daily intervention } \\
\text { protein and additional nutrients }\end{array}$ & $\begin{array}{l}\text { Rehab } \\
(\mathrm{Y} / \mathrm{N})\end{array}$ & Comparator/control \\
\hline Abe, 2016 & u & $\begin{array}{l}\text { Frail NH residents } \\
\text { a: MCT: } 13 / 12 \\
\text { b: LCT: } 13 / 12\end{array}$ & $\begin{array}{l}\text { MCT IG: } 85 \cdot 5 \text {, BMI } 18 \cdot 7 \\
\text { LCT IG: } 87 \cdot 7, \text { BMI } 18 \cdot 8 \\
\text { CG: } 86 \cdot 8, \text { BMI } 19 \cdot 6\end{array}$ & 3 months & $1 / d$, at $D$ & $\begin{array}{l}\text { a: MCT IG: } 6 \mathrm{~g} \mathrm{MCT}, 3.0 \mathrm{~g} \text { EAA, } \\
20 \mu \mathrm{g} \text { vit. D. } 334 \mathrm{~kJ}, 1.2 \mathrm{~g} \text { Leu/d. } \\
\text { b: LCT IG: } 6 \mathrm{~g} \text { LCT, } 3.0 \mathrm{~g} \mathrm{EAA}, \\
20 \mu \mathrm{g} \text { vit. D. } 351 \mathrm{~kJ}, 1.2 \mathrm{~g} \mathrm{Leu} / \mathrm{d}\end{array}$ & $\mathrm{N}$ & No treatment \\
\hline $\begin{array}{l}\text { Aleman- } \\
\text { Mateo, } \\
2012\end{array}$ & u & $\begin{array}{l}\text { Community sarcopenic } \\
20 / 20\end{array}$ & $\begin{array}{l}\text { IG: } 75 \cdot 4, \text { BMI } 26 \cdot 5 \\
\text { CG: } 76 \cdot 7, \text { BMI } 26 \cdot 1\end{array}$ & 3 months & $3 / d$, at $B F, L, D$ & $70 \mathrm{~g}$ ricotta* $1116 \mathrm{~kJ}, 15.7 \mathrm{~g} \mathrm{P} / \mathrm{d}$ & $\mathrm{N}$ & Usual diet and home visits \\
\hline $\begin{array}{l}\text { Aquiliani, } \\
2008\end{array}$ & $\mathrm{u}$ & $\begin{array}{l}\text { Outpatients, CHF } \\
22 / 22\end{array}$ & $\begin{array}{l}\text { IG: } 74 \cdot 5, \mathrm{BMI} 22 \cdot 5 \\
\text { CG: } 73 \cdot 1, \mathrm{BMI} 23 \cdot 2\end{array}$ & 2 months & $\begin{array}{l}\text { 2/d, at morning, } \\
\text { afternoon }\end{array}$ & $4 \mathrm{~g}$ EAA. $300 \mathrm{~kJ}, 8 \mathrm{~g}$ EAA, $2.5 \mathrm{~g} \mathrm{Leu} / \mathrm{d}$ & $\mathrm{N}$ & No treatment \\
\hline $\begin{array}{l}\text { Bakhitiari, } \\
2012\end{array}$ & $\mathrm{u}$ & $\begin{array}{l}\text { Community } \\
\text { a: Soya: } 25 / 25 \\
\text { b: TSP } 25 / 25\end{array}$ & $\begin{array}{l}\text { Soya IG: } 63 \cdot 8, \text { BMI } 28 \cdot 8 \\
\text { TSP IG: } 64 \cdot 6, \mathrm{BMI} 27.5 \\
\text { CG: } 64 \cdot 1, \mathrm{BMI} 28 \cdot 5\end{array}$ & 12 weeks & $1 / \mathrm{d}$ & $\begin{array}{l}\text { a: Soya IG: } 35 \mathrm{~g} \text { soya nut*. } 750 \mathrm{~kJ}, 13.8 \mathrm{~g} \mathrm{P} / \mathrm{d} \\
\text { b: TSP IG: } 35 \mathrm{~g} \text { TSP*. } 512 \mathrm{~kJ}, 18.2 \mathrm{~g} P / \mathrm{d}\end{array}$ & $\mathrm{N}$ & No treatment \\
\hline Bauer, 2015 & $\mathrm{~L}$ & $\begin{array}{l}\text { Sarcopenic } \\
184 / 196\end{array}$ & $\begin{array}{l}\text { IG: } 77 \cdot 3, \mathrm{BMI} 26 \cdot 0 \\
\text { CG: } 78 \cdot 1, \mathrm{BMI} 26 \cdot 2\end{array}$ & $13 w$ & $\begin{array}{l}\text { 2/d, before } B F, L \\
(I G \text { and } C G)\end{array}$ & $\begin{array}{l}20 \mathrm{~g} \text { Leu-enriched whey protein in water, } \\
20 \text { ug vit. D. } 1254 \mathrm{~kJ}, 41.4 \mathrm{~g} \mathrm{P}, 6 \mathrm{~g} \text { Leu/d }\end{array}$ & $\mathrm{N}$ & Placebo. 1254 kJ, $0 \mathrm{~g} \mathrm{P} / \mathrm{d}$ \\
\hline $\begin{array}{l}\text { Björkman, } \\
2012\end{array}$ & $\mathrm{u}$ & $\begin{array}{l}\mathrm{NH} \text { residents } \\
49 / 57\end{array}$ & $\begin{array}{l}\text { IG: } 84 \cdot 1, \mathrm{BMI} 24 \cdot 8 \\
\text { CG: } 83 \cdot 0, \mathrm{BMI} 24 \cdot 0\end{array}$ & 6 months & 3/d (IG and CG) & $\begin{array}{l}150 \mathrm{ml} \text { juice with whey protein*. } 1181 \mathrm{~kJ}, 20 \mathrm{~g} \\
\mathrm{P} / \mathrm{d}\end{array}$ & $\mathrm{Y}$ & $\begin{array}{l}150 \mathrm{ml} \mathrm{juice*} . \\
846 \mathrm{~kJ}, 0 \mathrm{~g} \mathrm{P} / \mathrm{d}\end{array}$ \\
\hline $\begin{array}{l}\text { Bonnefoy, } \\
2003\end{array}$ & u & $\begin{array}{l}\text { Retirement home frail } \\
30 / 27\end{array}$ & $\begin{array}{l}\text { IG: } 83 \text {, BMI } 27.13 \\
\text { CG: } 83 \text {, BMI } 27.32\end{array}$ & 9 months & $\begin{array}{l}\text { 2/d, at morning, } \\
\text { afternoon } \\
\text { (IG and CG) }\end{array}$ & $200 \mathrm{ml} \mathrm{ONS}^{*} .1686 \mathrm{~kJ}, 30 \mathrm{~g} \mathrm{P} / \mathrm{d}$ & $\mathrm{N}$ & $0 \mathrm{~kJ}$ placebo \\
\hline $\begin{array}{l}\text { Bonnefoy, } \\
2010\end{array}$ & $u$ & $\begin{array}{l}\text { Malnourished catabolic } \\
15 / 15\end{array}$ & $\begin{array}{l}\text { IG: } 82 \cdot 5, \text { BMI } 22 \cdot 6 \\
\text { CG: } 79 \cdot 4, \text { BMI } 23 \cdot 4\end{array}$ & 2 weeks & $3-5 / d$, at L, D & $\begin{array}{l}6.5-9 \mathrm{~g} \mathrm{P}(47.5 \% \text { BCAA). } 213-357 \mathrm{~kJ} \\
11.0-18.0 \mathrm{~g} \mathrm{P}, 3.6-6.0 \mathrm{~g} \text { Leu/d. Added to } \\
\text { food }\end{array}$ & $\mathrm{N}$ & Usual dietetic advice \\
\hline $\begin{array}{l}\text { Bouillanne, } \\
2013\end{array}$ & $u$ & $\begin{array}{l}\text { Rehabilitation } \\
\text { Malnourished/at risk } \\
30 / 36\end{array}$ & $\begin{array}{l}\text { IG: } 84 \cdot 1, \mathrm{BMI} 20 \cdot 7 \\
\text { CG: } 85 \cdot 7, \mathrm{BMI} 20 \cdot 9\end{array}$ & 6 weeks & $72 \% \mathrm{P}$ intake at $\mathrm{L}$ & $\begin{array}{l}\text { Protein-rich food and } 6 \mathrm{~g} \text { milk protein powder. } \\
\text { To achieve } 1.5 \mathrm{~g} \mathrm{P} / \mathrm{kg} \mathrm{BW} / \mathrm{d} \text { intake }\end{array}$ & $\mathrm{Y}$ & Usual diet, even spread $\mathrm{kJ}$ and $\mathrm{P}$ \\
\hline $\begin{array}{l}\text { Cameron, } \\
2011\end{array}$ & $u$ & $\begin{array}{l}\text { Undernourished fracture } \\
23 / 21\end{array}$ & $\begin{array}{l}\text { IG: } 83 \cdot 7, \mathrm{BMI} 21 \cdot 5 \\
\text { CG: } 87 \cdot 1, \mathrm{BMI} 21 \cdot 5\end{array}$ & $40 d$ & $1 / \mathrm{d}(\mathrm{IG}$ and $\mathrm{CG})$ & $\begin{array}{l}\text { Approximately } 235 \mathrm{ml} \text { milk- or milk and } \\
\text { soya-based ONS*. 1475-1986 kJ, } \\
17.6-21.3 \mathrm{~g} \mathrm{P} / \mathrm{d}\end{array}$ & Y & $\begin{array}{l}\mathrm{HP} \text { diet with HP milk. } 811 \mathrm{~kJ} \text {, } \\
11 \mathrm{~g} \mathrm{P} / \mathrm{d}\end{array}$ \\
\hline $\begin{array}{l}\text { Chapman, } \\
2009\end{array}$ & $\mathrm{u}$ & $\begin{array}{l}\text { Community } \\
\text { Undernourished } \\
13 / 13\end{array}$ & $\begin{array}{l}\text { IG: } 78 \cdot 5, \mathrm{BMI} 18 \cdot 7 \\
\text { CG: } 78 \cdot 5, \mathrm{BMI} 19\end{array}$ & 12 months & $1 / \mathrm{d}$, after meal & $237 \mathrm{ml}$ milk-based ONS*. $1986 \mathrm{~kJ}, 21.6 \mathrm{~g} \mathrm{P} / \mathrm{d}$ & $\mathrm{N}$ & Usual care \\
\hline Collins, 2005 & u & $\mathrm{NH}$, wound clinic $18 / 20$ & $\begin{array}{l}\text { IG: } 79 \cdot 2, \mathrm{BMI} 25 \cdot 5 \\
\text { CG: } 81 \cdot 0 \text {, BMI } 26 \cdot 4\end{array}$ & 4 weeks & $\begin{array}{l}\text { 3/d, with meals } \\
(I G \text { and } C G)\end{array}$ & $80 \mathrm{ml}, 8.4 \mathrm{~kJ}(2 \mathrm{kcal}) / \mathrm{ml}$ ONS ${ }^{*} .1995 \mathrm{~kJ}, 19.8 \mathrm{~g} \mathrm{P} / \mathrm{d}$ & $\mathrm{N}$ & $\begin{array}{l}80 \mathrm{ml}, 4.2 \mathrm{~kJ}(1 \mathrm{kcal}) / \mathrm{ml} \mathrm{ONS} \text {. } \\
1050 \mathrm{~kJ}, 8.8 \mathrm{~g} \mathrm{P} / \mathrm{d}\end{array}$ \\
\hline $\begin{array}{l}\text { Dal Negro, } \\
2010\end{array}$ & $u$ & $\begin{array}{l}\text { Outpatients sarcopenic } \\
\text { COPD } \\
16 / 16\end{array}$ & $\begin{array}{l}\text { IG: } 75 \text {, BMI } 20.2 \\
\text { CG: } 75, \text { BMI } 20 \cdot 2\end{array}$ & 12 weeks & $2 / d(I G$ and $C G)$ & $4 \mathrm{~g}$ EAA. $8 \mathrm{~g}$ EAA $/ \mathrm{d}$ & $\mathrm{N}$ & Placebo \\
\hline $\begin{array}{l}\text { Dal Negro, } \\
2012\end{array}$ & u & $\begin{array}{l}\text { Outpatients sarcopenic } \\
\text { COPD } 44 / 44\end{array}$ & $\begin{array}{l}\text { IG: } 75, \mathrm{BMI} 20 \cdot 0 \\
\text { CG: } 73, \mathrm{BMI} 20 \cdot 1\end{array}$ & 12 weeks & $\begin{array}{l}2 / \mathrm{d} \text {, at morning and } \\
\text { afternoon (IG and } \mathrm{CG})\end{array}$ & $4 \mathrm{~g}$ EAA. $183 \mathrm{~kJ}, 8 \mathrm{~g}$ EAA, $2.5 \mathrm{~g}$ Leu/d & $\mathrm{N}$ & Placebo \\
\hline $\begin{array}{l}\text { Dangour, } \\
2011\end{array}$ & u & $\begin{array}{l}\text { Community } \\
\text { Low-middle SES } \\
502 / 504\end{array}$ & $\begin{array}{l}\text { All participants } 65-67 \cdot 9 \\
\text { IG: BMI } 28 \cdot 2 \\
\text { CG: BMI } 28 \cdot 6\end{array}$ & 24 months & $1 / \mathrm{d}$ & $\begin{array}{l}50 \mathrm{~g} \text { vegetable powder* and } 50 \mathrm{~g} \text { low lactose } \\
\text { milk powder*. } 1685 \mathrm{~kJ}, 15.5 \mathrm{~g} \mathrm{P} / \mathrm{d}\end{array}$ & $\mathrm{N}$ & No treatment \\
\hline Dreyer, 2013 & $\mathrm{u}$ & $\begin{array}{l}\text { Post-surgical, TKA } \\
21 / 19\end{array}$ & $\begin{array}{l}\text { IG: } 68, \text { BMI } 34 \\
\text { CG: } 70 \text {, BMI } 29\end{array}$ & 3 weeks & $\begin{array}{l}\text { 2/d, at morning and } \\
\text { afternoon (IG and CG) }\end{array}$ & $20 \mathrm{~g}$ EAA. $7 \cdot 2 \mathrm{~g} \mathrm{Leu} / \mathrm{d}$ & $\mathrm{Y}$ & $2 / \mathrm{d}, 20 \mathrm{~g}$ alanine \\
\hline $\begin{array}{l}\text { Espaulella, } \\
2000\end{array}$ & L & $\begin{array}{l}\text { Inpatients, fracture } \\
85 / 86\end{array}$ & $\begin{array}{l}\text { IG: } 82 \cdot 4, \mathrm{BMI} 25 \cdot 4 \\
\text { CG: } 82 \cdot 7, \mathrm{BMI} 25 \cdot 4\end{array}$ & $\begin{array}{l}60 \mathrm{~d}+6 \text { months } \\
\text { F/U }\end{array}$ & $1 / \mathrm{d}(I G$ and $C G)$ & $200 \mathrm{ml} \mathrm{ONS}^{*} / \mathrm{d} .623 \mathrm{~kJ}, 20 \mathrm{~g} \mathrm{P} / \mathrm{d}$ & $\mathrm{N}$ & $200 \mathrm{ml}$ placebo. $648 \mathrm{~kJ}, 0 \mathrm{~g} \mathrm{P} / \mathrm{d}$ \\
\hline $\begin{array}{l}\text { Fiatarone, } \\
1994\end{array}$ & u & $\begin{array}{l}\mathrm{NH} \text {, frail } \\
24 / 26\end{array}$ & $\begin{array}{l}\text { IG: } 85 \cdot 7, \text { BMI } 25 \cdot 4 \\
\text { CG: } 89 \cdot 2 \text {, BMI } 25 \cdot 8\end{array}$ & 10 weeks & $1 / \mathrm{d}$, at $P M(I G$ and $C G)$ & $240 \mathrm{ml}$ soya-based ONS*. $1505 \mathrm{~kJ}, 15.3 \mathrm{~g} \mathrm{P} / \mathrm{d}$ & $\mathrm{N}$ & $240 \mathrm{ml}$ placebo. $17 \mathrm{~kJ} / \mathrm{d}$ \\
\hline $\begin{array}{l}\text { Gariballa, } \\
2007\end{array}$ & L & $\begin{array}{l}\text { Hospital } \\
106 / 119\end{array}$ & $\begin{array}{l}\text { IG: } 75 \cdot 9, \mathrm{BMI} 25 \cdot 2 \\
\text { CG: } 75 \cdot 3, \mathrm{BMI} 25 \cdot 2\end{array}$ & $\begin{array}{l}6 \text { weeks }+6 \\
\text { months } F / U\end{array}$ & $\begin{array}{l}\text { 2/d, at morning and } \\
\text { afternoon (IG and } C G)\end{array}$ & $200 \mathrm{ml} O \mathrm{ONS}^{*} .4160 \mathrm{~kJ}, 49.8 \mathrm{~g} \mathrm{P} / \mathrm{d}$ & $\mathrm{N}$ & $251 \mathrm{~kJ}$ placebo \\
\hline Kim, 2012 & u & $\begin{array}{l}\text { Community sarcopenic } \\
39 / 39\end{array}$ & $\begin{array}{l}\text { IG: } 79 \cdot 2, \mathrm{BMI} 18 \cdot 9 \\
\text { CG: } 78 \cdot 7, \mathrm{BMI} 18 \cdot 8\end{array}$ & 3 months & $2 / \mathrm{d}$, in milk/water & $3 \mathrm{~g}$ EAA. $6 \mathrm{~g}$ EAA, $2.5 \mathrm{~g} \mathrm{Leu} / \mathrm{d}$ & $\mathrm{N}$ & Health education classes \\
\hline Kim, 2013 & $\mathrm{u}$ & $\begin{array}{l}\text { Community, frail } \\
43 / 44\end{array}$ & $\begin{array}{l}\text { IG: } 78.9 \\
\text { CG: } 78.4\end{array}$ & 12 weeks & $2 / d$ & $200 \mathrm{ml} \mathrm{ONS}^{*} .1672 \mathrm{~kJ}, 25 \mathrm{~g} \mathrm{P} / \mathrm{d}$ & $\mathrm{N}$ & Home visits \\
\hline Lauque, 2000 & $\mathrm{u}$ & $\begin{array}{l}\mathrm{NH}, \text { malnourished } \\
19 / 22\end{array}$ & $\begin{array}{l}\text { IG: } 84 \cdot 6, \mathrm{BMI} 22 \cdot 3 \\
\text { CG: } 84 \cdot 7, \mathrm{BMI} 21 \cdot 8\end{array}$ & $60 d$ & $1-2 / d$, with meals & $\begin{array}{l}150-200 \mathrm{ml} \text { milk-, whey- or milk and pea } \\
\text { protein-based } \\
\text { ONS*. } 1254-2090 \mathrm{~kJ}, 24-39 \mathrm{~g} \mathrm{P} / \mathrm{d}\end{array}$ & $\mathrm{N}$ & No treatment \\
\hline
\end{tabular}




\section{N British Journal of Nutrition}

Table 1. Continued

\begin{tabular}{|c|c|c|c|c|c|c|c|c|}
\hline $\begin{array}{l}\text { First author, } \\
\text { year }\end{array}$ & $\begin{array}{l}\text { Study } \\
\text { ROB }\end{array}$ & $\begin{array}{l}\text { Population sample size } \\
\text { (IG/CG) }\end{array}$ & $\begin{array}{l}\text { Mean age (years), } \\
\text { baseline BMI }\left(\mathrm{kg} / \mathrm{m}^{2}\right)\end{array}$ & Study length & Ingestion details & $\begin{array}{l}\text { Dose. Total daily intervention } \\
\text { protein and additional nutrients }\end{array}$ & $\begin{array}{l}\text { Rehab } \\
(\mathrm{Y} / \mathrm{N})\end{array}$ & Comparator/control \\
\hline Lauque, 2004 & u & $\begin{array}{l}\text { Hospital, AD care } \\
46 / 45\end{array}$ & $\begin{array}{l}\text { IG: } 79 \cdot 5, \mathrm{BMI} 22 \cdot 2 \\
\text { CG: } 78 \cdot 1, \mathrm{BMI} 22 \cdot 6\end{array}$ & $\begin{array}{l}3 \text { months } \\
+3 \text { months } F / U\end{array}$ & $1-2 / d$ & $\begin{array}{l}\text { 150-200 ml milk- or milk and pea } \\
\text { protein-based ONS } \\
1254-2090 \mathrm{~kJ}, 11-34 \mathrm{~g} \mathrm{P} / \mathrm{d}\end{array}$ & $\mathrm{N}$ & Usual care \\
\hline $\begin{array}{l}\text { Leenders, } \\
2011\end{array}$ & $\mathrm{u}$ & $\begin{array}{l}\text { Type } 2 \text { diabetic } \\
30 / 30\end{array}$ & $\begin{array}{l}\text { IG: } 71, \mathrm{BMI} 27 \cdot 4 \\
\text { CG: } 71, \mathrm{BMI} 27 \cdot 2\end{array}$ & 6 months & $\begin{array}{l}\text { 3/d, at BF, L, D } \\
\text { (IG and CG) }\end{array}$ & $2.5 \mathrm{~g} \mathrm{Leu} .7 .5 \mathrm{~g} \mathrm{Leu} / \mathrm{d}$ & $\mathrm{N}$ & 3/d, wheat flour placebo \\
\hline $\begin{array}{l}\text { McMurdo, } \\
2009\end{array}$ & $\mathrm{u}$ & $\begin{array}{l}\text { Hospital undernourished } \\
127 / 126\end{array}$ & $\begin{array}{l}\text { IG: } 81 \cdot 4, \mathrm{BMI} 21 \cdot 2 \\
\text { CG: } 82 \cdot 2, \mathrm{BMI} 21 \cdot 2\end{array}$ & 16 weeks & $2 / d(I G$ and $C G)$ & $\begin{array}{l}200 \mathrm{ml} \text { milk-based ONS*. } 2520 \mathrm{~kJ} \\
40 \mathrm{~g} \mathrm{P} / \mathrm{d}\end{array}$ & $\mathrm{Y}$ & $\begin{array}{l}200 \mathrm{ml} \text { skimmed milk ONS*} \\
840 \mathrm{~kJ}, 12.4 \mathrm{~g} \mathrm{P} / \mathrm{d}\end{array}$ \\
\hline Miller, 2006 & $\mathrm{u}$ & $\begin{array}{l}\text { Hospital, fracture } \\
25 / 26\end{array}$ & $\begin{array}{l}\text { IG: } 83 \cdot 5, \mathrm{BMI} 21 \cdot 9 \\
\text { CG: } 83 \cdot 1, \mathrm{BMI} 22 \cdot 1\end{array}$ & 6 weeks & 4/d, during Medpass & $\begin{array}{l}145-200 \mathrm{ml} \text { milk-based ONS } \\
3654-5040 \mathrm{~kJ}, 34 \cdot 8-48 \mathrm{~g} \mathrm{P} / \mathrm{d}^{*}\end{array}$ & $\mathrm{Y}$ & Attention control and home visits \\
\hline Myint, 2013 & $\mathrm{u}$ & $\begin{array}{l}\text { Hospital, fracture } \\
61 / 61\end{array}$ & $\begin{array}{l}\text { IG: } 80 \cdot 9, \mathrm{BMI} 20.2 \\
\text { CG: } 81.7, \mathrm{BMI} 21 \cdot 2\end{array}$ & $28 \mathrm{~d}$ & $2 / \mathrm{d}$ & $\begin{array}{l}\text { Approximately } 240 \mathrm{ml} \text { milk-, whey- or milk and } \\
\text { soya-based ONS*. } 2090 \mathrm{~kJ}, \\
14-19 \mathrm{~g} \mathrm{P} / \mathrm{d} .20-25 \mu \mathrm{g} \text { vit. D, } \\
1.2 \mathrm{~g} \mathrm{Ca} / \mathrm{d}\end{array}$ & Y & $20-25 \mu \mathrm{g}$ vit. D, $1.2 \mathrm{~g} \mathrm{Ca} / \mathrm{d}$ \\
\hline $\mathrm{Ng}, 2015$ & $\mathrm{~L}$ & $\begin{array}{l}\text { Community } \\
\text { Pre-frail and frail } \\
49 / 50\end{array}$ & $\begin{array}{l}\text { IG: } 69 \cdot 7, \mathrm{BMI} 24 \cdot 3 \\
\text { CG: } 70 \cdot 1, \mathrm{BMI} 23 \cdot 6\end{array}$ & 24 weeks & $1 / \mathrm{d}$ & $\begin{array}{l}200 \mathrm{ml} \text { milk-based ONS. } 1260 \mathrm{~kJ}, 12 \mathrm{~g} \mathrm{P} / \mathrm{d} \text {. } \\
1 \mathrm{mg} \text { folate, } 29 \mathrm{mg} \mathrm{Fe}, 200 \mu \mathrm{g} \text { vit. } \mathrm{B}_{12} \\
200 \mathrm{mg} \text { vit. } \mathrm{B}_{6}, 5 \mu \mathrm{g} \text { vit. D, } 0.6 \mathrm{~g} \mathrm{Ca} / \mathrm{d}\end{array}$ & $\mathrm{N}$ & $\begin{array}{l}\text { Artificially sweetened equal } \\
\text { volume placebo }\end{array}$ \\
\hline Payette, 2002 & $\mathrm{u}$ & $\begin{array}{l}\text { Community, frail } \\
\text { undernourished } \\
45 / 46\end{array}$ & $\begin{array}{l}\text { IG: } 81 \cdot 6, \mathrm{BMI} 20 \cdot 1 \\
\text { CG: } 78 \cdot 6, \mathrm{BMI} 20 \cdot 1\end{array}$ & 16 weeks & $2 / d$ & $\begin{array}{l}235 \mathrm{ml} \text { milk and soya-based ONS*. 2090- } \\
2096 \mathrm{~kJ}, 18 \cdot 8-26.6 \mathrm{~g} \mathrm{P} / \mathrm{d}\end{array}$ & $\mathrm{N}$ & Monthly home visits \\
\hline $\begin{array}{l}\text { Persson, } \\
2007\end{array}$ & $\mathrm{u}$ & $\begin{array}{l}\text { Hospital } \\
\text { Malnutrition risk } \\
51 / 57\end{array}$ & $\begin{array}{l}\text { IG: } 85 \text {, BMI } 19 \cdot 8 \\
\text { CG: } 85, \text { BMI } 17.4\end{array}$ & 4 months & $1-2 / d$ & $\begin{array}{l}200 \mathrm{ml} \mathrm{ONS}^{\star} .711-2006 \mathrm{~kJ}, 8-20 \mathrm{~g} \mathrm{P.} 200 \mu \mathrm{g} \\
\quad \text { folate, } 3 \mu \mathrm{g} \text { vit. } \mathrm{B}_{12}, 5 \mu \mathrm{g} \text { vit. D, } 12 \mathrm{mg} \mathrm{Zn/d}\end{array}$ & $\mathrm{N}$ & Written dietetic advice \\
\hline $\begin{array}{l}\text { Rondanelli, } \\
2011\end{array}$ & $\mathrm{u}$ & $\begin{array}{l}\mathrm{NH} \\
20 / 21\end{array}$ & $\begin{array}{l}\text { IG: } 83 \cdot 5, \mathrm{BMI} 21 \cdot 8 \\
\text { CG: } 79 \cdot 9, \mathrm{BMI} 22 \cdot 1\end{array}$ & 8 weeks & $\begin{array}{l}\text { 2/d, at morning and } \\
\text { afternoon } \\
\text { (IG and CG) }\end{array}$ & $4 \mathrm{~g}$ EAA. $300 \mathrm{~kJ}, 8 \mathrm{~g}$ EAA, $2.5 \mathrm{~g} \mathrm{Leu} / \mathrm{d}$ & $\mathrm{N}$ & $4 \mathrm{~g}$ isoenergetic placebo \\
\hline $\begin{array}{l}\text { Rosendahl, } \\
2006\end{array}$ & $\mathrm{~L}$ & $\begin{array}{l}\text { Residential care, frail } \\
50 / 50\end{array}$ & $\begin{array}{l}\text { IG: } 82 \cdot 9, \mathrm{BMI} 24 \cdot 9 \\
\text { CG: } 85 \cdot 6, \mathrm{BMI} 24 \cdot 5\end{array}$ & $\begin{array}{l}3 \text { months } \\
\quad+3 \text { months } F / U\end{array}$ & $\begin{array}{l}\text { 5/2w, after OT } \\
\text { (IG and CG) }\end{array}$ & $200 \mathrm{ml}$ milk-based ONS*. $816 \mathrm{~kJ}, 14.8 \mathrm{~g} \mathrm{P} / \mathrm{d}$ & $\mathrm{N}$ & Placebo. $382 \mathrm{~kJ}, 0.4 \mathrm{~g} \mathrm{P} / \mathrm{d}$ \\
\hline $\begin{array}{l}\text { Schurch, } \\
1998\end{array}$ & $\mathrm{u}$ & $\begin{array}{l}\text { Hospital, fracture } \\
41 / 41\end{array}$ & $\begin{array}{l}\text { IG: } 81 \cdot 1, \text { BMI } 24 \cdot 2 \\
\text { CG: } 80 \cdot 2 \text {, BMI } 24 \cdot 4\end{array}$ & 6 months & $5 / w$ (IG and CG) & $200 \mathrm{ml} \mathrm{ONS}^{*} .1050 \mathrm{~kJ}, 20 \mathrm{~g}$ P/d. $1 \times 5 \mathrm{mg}$ vit. D & $\mathrm{Y}$ & $\begin{array}{l}\text { Isoenergetic placebo. } \\
1 \times 5 \mathrm{mg} \text { vit. } D\end{array}$ \\
\hline $\begin{array}{l}\text { Smoliner, } \\
2008\end{array}$ & $\mathrm{u}$ & $\begin{array}{l}\mathrm{NH}, \text { malnourished or } \\
\text { at risk } \\
22 / 30\end{array}$ & $\begin{array}{l}\text { IG: } 82 \cdot 2, \text { BMI } 21 \cdot 6 \\
\text { CG: } 84, \text { BMI } 22 \cdot 5\end{array}$ & 12 weeks & 1 ONS/d & $\begin{array}{l}\text { Milk-based ONS* and enriched foods. } 1254 \mathrm{~kJ} \text {, } \\
20 \mathrm{~g} \mathrm{P} / \mathrm{d} \text { from ONS and } 5 \mathrm{~g} / 100 \mathrm{ml} \text { from } \\
\text { soups } / \text { sauces }\end{array}$ & $\mathrm{N}$ & Usual care \\
\hline $\begin{array}{l}\text { Sugawara, } \\
2012\end{array}$ & $u$ & $\begin{array}{l}\text { Stable COPD } \\
18 / 18\end{array}$ & $\begin{array}{l}\text { IG: } 77 \cdot 4 \\
\text { CG: } 77 \cdot 1\end{array}$ & 3 months & $2 / d$ & $200 \mathrm{ml}$ whey-containing ONS $\mathrm{OS}^{\star} .1672 \mathrm{~kJ}, 20 \mathrm{~g} \mathrm{P} / \mathrm{d}$ & $\mathrm{Y}$ & Usual care and diet instructions \\
\hline Tieland, 2012 & $\mathrm{~L}$ & Community, frail $34 / 31$ & $\begin{array}{l}\text { IG: } 78, \text { BMI } 27 \\
\text { CG: } 81, \text { BMI } 26 \cdot 2\end{array}$ & 24 weeks & $2 / \mathrm{d}$, at $\mathrm{BF}, \mathrm{L}$ & $\begin{array}{l}250 \mathrm{ml} \mathrm{milk-based} \mathrm{ONS}{ }^{*} .777 \mathrm{~kJ} \\
30 \mathrm{~g} \mathrm{P} / \mathrm{d}\end{array}$ & $\mathrm{N}$ & $2 / \mathrm{d}, 250 \mathrm{ml} 0 \mathrm{~g}$ protein placebo \\
\hline Volkert, 1996 & $\mathrm{u}$ & $\begin{array}{l}\text { Hospital undernourished } \\
\text { a: Compliers } 11 / 37 \\
\text { b: Non-compliers } 9 / 37\end{array}$ & $\begin{array}{l}\text { a: IG compliers: } 84 \cdot 5 \text {, } \\
\text { BMI } 19 \cdot 8 \\
\text { b: IG non-compliers: } \\
88 \cdot 7, \text { BMI } 19 \cdot 1 \\
\text { CG: } 84 \cdot 0, \text { BMI } 19 \cdot 3\end{array}$ & $\begin{array}{l}\text { Approximately } \\
3 \text { weeks } \\
\text { hospital + } \\
6 \text { months F/U }\end{array}$ & $\begin{array}{l}\text { 2/d, at morning, } \\
\text { afternoon. After } \\
\text { D/C, } 1 / \mathrm{d}\end{array}$ & $\begin{array}{l}200 \mathrm{ml} \text { ONS*. } 2022-2102 \mathrm{~kJ}, 30 \mathrm{~g} \mathrm{P} / \mathrm{d} \text {. } \\
\text { After D/C, } 200 \mathrm{ml} \mathrm{ONS} \text {. } 1000-1080 \mathrm{~kJ} \text {, } \\
16 \mathrm{~g} \mathrm{P} / \mathrm{d}\end{array}$ & $\mathrm{Y}$ & Usual care \\
\hline Yamada, 2015 & u & $\begin{array}{l}\text { Community } \\
79 / 71\end{array}$ & $\begin{array}{l}\text { IG: } 76 \cdot 3, \mathrm{BMI} 22 \cdot 3 \\
\text { CG: } 75 \cdot 8, \mathrm{BMI} 22 \cdot 7\end{array}$ & 6 months & $1 / d$ & $125 \mathrm{ml}$ milk-based ONS*. $836 \mathrm{~kJ}, 10 \mathrm{~g} \mathrm{P} / \mathrm{d}$ & $\mathrm{Y}$ & No treatment \\
\hline Zak, 2009 & L & $\begin{array}{l}\mathrm{NH} / \text { community } \\
\text { Frail } 23 / 23\end{array}$ & $\begin{array}{l}\text { IG: } 78 \cdot 3, \text { BMI } 25 \cdot 2 \\
\text { CG: } 81 \cdot 1, \text { BMI } 25 \cdot 2\end{array}$ & 7 weeks & $\begin{array}{l}\text { 5/w, before exercise } \\
\text { (IG and CG) }\end{array}$ & $200 \mathrm{ml}$ milk-based ONS*. $1254 \mathrm{~kJ}, 12 \mathrm{~g} \mathrm{P} / \mathrm{d}$ & Y & $200 \mathrm{ml}$ placebo. $171 \mathrm{~kJ}, 0.1 \mathrm{~g} \mathrm{P} / \mathrm{d}$ \\
\hline
\end{tabular}

ROB, risk of bias; U, unclear; L, low or lunch; IG, intervention group; CG, control group; NH, nursing home; MCT, medium-chain TAG; LCT, long-chain TAG; CHF, chronic heart failure; TSP, textured soya protein; COPD, chronic obstructive pulmonary disease; SES, socioeconomic status; TKA, total knee arthroplasty; AD, Alzheimer's disease; F/U, follow up; D, dinner; BF, breakfast; P, protein; OT, occupational therapy; D/C, discharge; EAA, essential amino acids; vit., vitamin; Leu, leucine; ONS, oral nutritional supplement; BCAA, branched chain amino acids; BW, body weight; HP, high protein.

* Intervention (ONS, protein-rich food, etc.) provides additional vitamins and minerals, in addition to protein and $\mathrm{kJ}$. 
Effect of protein and amino acid supplements

Study name

Aleman-Mateo et al., 2012

Bakhitiari et al., 2012a

Bakhitiari et al., 2012b

Bjorkman et al., 2012

Bonnefoy et al., 2003

Bonnefoy et al., 2010

Bouillanne et al., 2013

Chapman et al., 2009

Dal Negro et al., 2010

Dal Negro et al., 2012

Kim et al., 2012

Lauque et al., 2004

Leenders et al., 2011

Smoliner et al., 2008

Sugawara et al., 2012

Tieland et al., 2012

Yamada et al., 2015
Statistics for each study

\begin{tabular}{|c|c|c|c|c|}
\hline \multirow[b]{2}{*}{$\begin{array}{l}\text { Std diff } \\
\text { in means }\end{array}$} & \\
\hline & $\mathrm{SE}$ & $\begin{array}{l}\text { Lower } \\
\text { limit }\end{array}$ & $\begin{array}{l}\text { Upper } \\
\text { limit }\end{array}$ & $P$ \\
\hline 0.00 & 0.32 & -0.62 & 0.62 & 1.000 \\
\hline 0.35 & 0.29 & -0.20 & 0.91 & 0.214 \\
\hline 0.31 & 0.28 & -0.25 & 0.87 & 0.275 \\
\hline 0.10 & 0.22 & -0.34 & 0.53 & 0.656 \\
\hline 0.78 & 0.32 & 0.15 & 1.41 & 0.015 \\
\hline-0.13 & 0.47 & -1.04 & 0.79 & 0.787 \\
\hline 0.68 & 0.26 & 0.17 & $1 \cdot 18$ & 0.009 \\
\hline$-0 \cdot 10$ & 0.41 & -0.90 & 0.70 & 0.803 \\
\hline $1 \cdot 17$ & 0.38 & 0.42 & 1.92 & 0.002 \\
\hline-0.04 & 0.21 & -0.46 & 0.38 & 0.84 \\
\hline 0.07 & 0.23 & -0.39 & 0.52 & 0.769 \\
\hline 0.35 & 0.23 & -0.09 & 0.79 & 0.121 \\
\hline 0.02 & 0.26 & -0.50 & 0.54 & 0.93 \\
\hline 0.00 & 0.28 & -0.55 & 0.55 & 1.000 \\
\hline 0.29 & 0.36 & -0.42 & 1.00 & 0.428 \\
\hline 0.01 & 0.25 & -0.47 & 0.50 & 0.95 \\
\hline 0.15 & 0.17 & -0.17 & 0.48 & 0.35 \\
\hline 0.21 & 0.07 & 0.07 & 0.35 & 0.00 \\
\hline
\end{tabular}

Std diff in means and $95 \% \mathrm{Cl}$

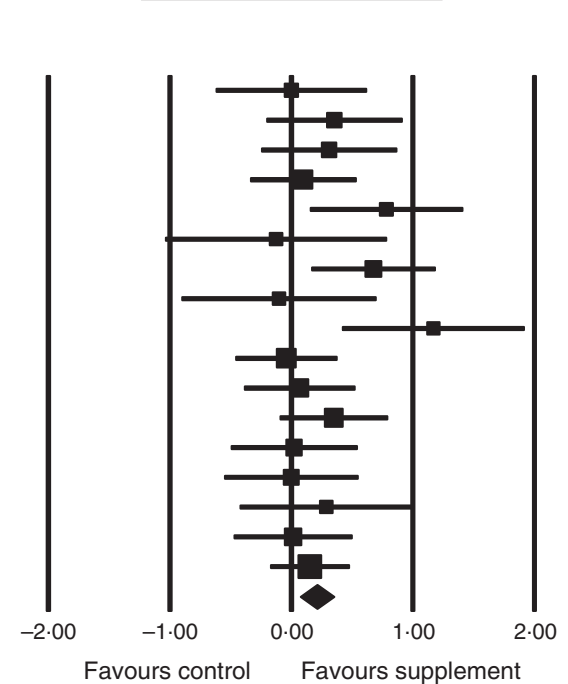

Relative

weight

4.44

$5 \cdot 32$

$5 \cdot 34$

8.05

4.33

$2 \cdot 18$

$6 \cdot 24$

$2 \cdot 78$

$3 \cdot 14$

$8 \cdot 61$

$7 \cdot 49$

7.85

6.04

$5 \cdot 47$

$3 \cdot 47$

$6 \cdot 73$

$12 \cdot 51$

Fig. 2. Forest plot of effect of supplements on fat-free mass. Random effects model. Heterogeneity: $P$ 15.1, $P=0.28$.

Study name

Abe et al., $2016 a$

Abe et al., 2016b

Aleman-Mateo et al., 2012

Aquiliani et al. 2008

Bauer et al., 2015

Bjorkman et al., 2012

Bouillanne et al., 2013

Cameron et al., 2011

Chapman et al., 2009

Dal Negro et al., 2012

Dreyer et al., 2013

Fiatarone et al., 1994

Kim and Lee, 2013

Kim et al., 2012

Lauque et al., 2000

Leenders et al., 2011

McMurdo et al., 2009

Miller et al., 2006

Myint et al., 2013

$\mathrm{Ng}$ et al., 2015

Payette et al., 2002

Persson et al., 2007

Rondanelli et al., 2011

Rosendahl et al., 2006

Schurch et al., 1998

Smoliner et al., 2008

Sugawara et al., 2012

Tieland et al., 2012

Zak et al., 2009

\begin{tabular}{|c|c|c|c|c|}
\hline \multirow[b]{2}{*}{$\begin{array}{c}\text { Std diff } \\
\text { in means }\end{array}$} & \multicolumn{2}{|c|}{ Statistics for each study } & \multirow[b]{2}{*}{$\begin{array}{l}\text { Upper } \\
\text { limit }\end{array}$} & \multirow[b]{2}{*}{$P$} \\
\hline & SE & $\begin{array}{l}\text { Lower } \\
\text { limit }\end{array}$ & & \\
\hline 1.44 & 0.47 & 0.52 & $2 \cdot 37$ & 0.002 \\
\hline 0.53 & 0.44 & -0.34 & 1.40 & 0.234 \\
\hline 0.17 & 0.32 & -0.45 & 0.80 & 0.583 \\
\hline 0.67 & 0.34 & 0.02 & 1.33 & 0.045 \\
\hline 0.07 & $0 \cdot 10$ & $-0 \cdot 13$ & 0.28 & 0.479 \\
\hline 0.12 & 0.25 & -0.37 & 0.62 & 0.621 \\
\hline-0.28 & 0.25 & -0.77 & 0.22 & 0.278 \\
\hline-0.07 & 0.30 & -0.66 & 0.52 & 0.821 \\
\hline 0.56 & 0.42 & -0.25 & 1.38 & 0.176 \\
\hline $1 \cdot 86$ & 0.26 & $1 \cdot 36$ & $2 \cdot 36$ & 0.000 \\
\hline $0 \cdot 18$ & 0.38 & -0.57 & 0.93 & 0.632 \\
\hline-0.31 & 0.29 & -0.89 & 0.27 & 0.293 \\
\hline-0.09 & 0.22 & -0.51 & 0.34 & 0.694 \\
\hline 0.72 & 0.24 & 0.25 & $1 \cdot 19$ & 0.003 \\
\hline 0.23 & 0.35 & -0.46 & 0.91 & 0.521 \\
\hline 0.07 & 0.27 & -0.45 & 0.59 & 0.793 \\
\hline 0.42 & 0.15 & 0.14 & 0.71 & 0.004 \\
\hline-0.12 & 0.28 & -0.67 & 0.43 & 0.675 \\
\hline-0.14 & 0.19 & -0.51 & 0.22 & 0.435 \\
\hline 0.47 & 0.20 & 0.07 & 0.87 & 0.022 \\
\hline-0.15 & 0.22 & -0.58 & 0.28 & 0.498 \\
\hline 0.29 & 0.27 & -0.25 & 0.83 & 0.286 \\
\hline 1.07 & 0.33 & 0.41 & $1 \cdot 72$ & 0.001 \\
\hline-0.05 & 0.29 & -0.61 & 0.52 & 0.873 \\
\hline 0.40 & 0.25 & -0.09 & 0.90 & 0.112 \\
\hline 0.13 & 0.28 & -0.42 & 0.69 & 0.631 \\
\hline 0.42 & 0.36 & -0.29 & $1 \cdot 14$ & 0.245 \\
\hline 0.00 & 0.25 & -0.49 & 0.49 & 1.000 \\
\hline 0.03 & 0.32 & -0.59 & 0.65 & 0.916 \\
\hline 0.27 & 0.09 & 0.10 & 0.44 & 0.002 \\
\hline
\end{tabular}

Std diff in means and $95 \% \mathrm{Cl}$

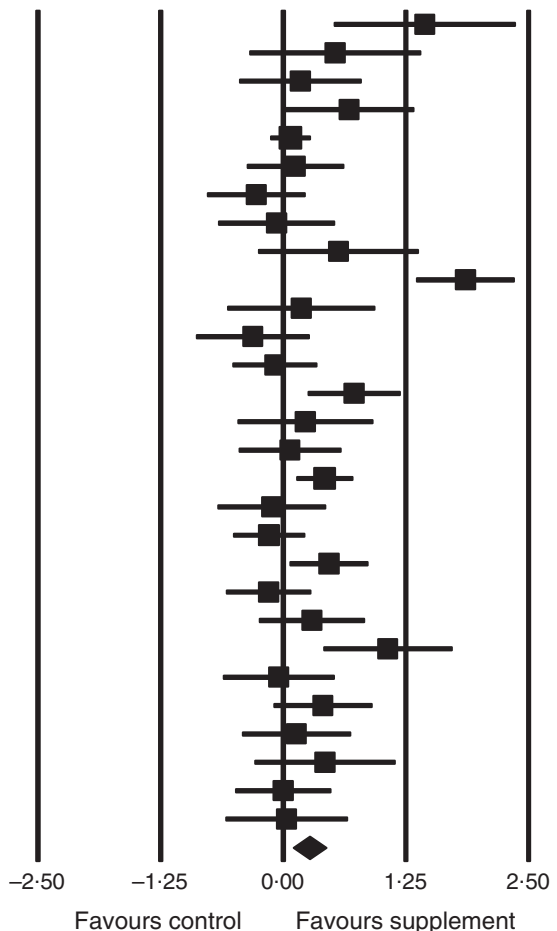

Relative

weight

$2 \cdot 05$

$2 \cdot 21$

$3 \cdot 13$

$2 \cdot 98$

$5 \cdot 10$

3.73

$3 \cdot 70$

$3 \cdot 26$

2.38

$3 \cdot 69$

$2 \cdot 61$

3.33

$4 \cdot 05$

3.84

2.85

$3 \cdot 60$

$4 \cdot 75$

$3 \cdot 46$

$4 \cdot 38$

$4 \cdot 20$

$4 \cdot 04$

3.51

$2 \cdot 99$

3.38

3.70

$3 \cdot 45$

$2 \cdot 74$

$3 \cdot 13$

Fig. 3. Forest plot of effect of supplements on muscle strength. Random effects model. Heterogeneity: $P 68 \cdot 4, P<0.01$.

\section{Muscle strength}

A total of twenty-eight studies reported measurements of muscle strength (analysed $n$ 1940), with handgrip strength the most common assessment, followed by leg quadriceps strength. The remaining test measures included were hip and knee, bicep, knee and cycling strength. One study ${ }^{(58)}$ was excluded from analysis, as its SMD results were more than $2 \mathrm{sD}$ from the mean. The effect of supplementation on muscle strength in the twenty-eight studies (Fig. 3) was found to be statistically significant with a small effect size (SMD 0.27; $95 \%$ CI 0.10, 0.44, $P<0.01$, significant heterogeneity, $\left.I^{2}=68.4, \quad P<0.01\right)$. Five studies had low $\operatorname{ROB}^{(43,46,56,70,74)}$ and no significant effect on muscle strength was found; however, a small effect was seen in studies with unclear ROB (SMD 0.31; 95\% CI $0 \cdot 10$, $0.52, P<0.01$, significant heterogeneity, $\left.I^{2}=72 \cdot 0, \quad P<0.01\right)$. 
Study name

Abe et al., 2016a

Abe et al., 2016b

Aquiliani et al., 2008

Bauer et al., 2015

Bjorkman et al., 2012

Bonnefoy et al., 2003

Bouillanne et al., 2013

Cameron et al., 2011

Chapman et al, 2009

Collins et al., 2005

Dal Negro et al., 2010

Dal Negro et al., 2012

Dangour et al., 2011

Dreyer et al., 2013

Espaulella et al., 2000

Fiatarone et al., 1994

Gariballa and Forster, 2007

Kim and Lee, 2013

Kim et al., 2012

Lauque et al., 2004

McMurdo et al., 2009

Miller et al., 2006

Myint et al., 2013

$\mathrm{Ng}$ et al., 2015

Payette et al., 2002

Persson et al., 2007

Rondanelli et al., 2011

Rosendahl et al., 2006

Schurch et al., 1998

Smoliner et al., 2008

Sugawara et al., 2012

Tieland et al., 2012

Volkert et al., 1996a

Volkert et al., 1996b

Yamada et al., 2015

Zak et al., 2009
Statistics for each study

$\begin{array}{cccc} & \begin{array}{c}\text { Lower } \\ \text { SE }\end{array} & \begin{array}{c}\text { Upper } \\ \text { limit }\end{array} & \\ \text { limit } & \\ 0.54 & 0.07 & 2.20 & 0.036 \\ 0.52 & -0.76 & 1.28 & 0.615\end{array}$

Std diff
in mean

$1 \cdot 14$

0.26

0.64

0.06

0.00

$-0.05$

0.40

0.59

0.32

$-0.05$

1.31

1.31

0.05

0.07

$-0.06$

0.21

0.37

0.40

0.68

0.08

0.45

0.15

0.03

$-0.08$

$-0.01$

0.76

0.59

$-0.12$

$-0 \cdot 41$

0.25

0.79

0.43

0.41

$-0.55$

$-0.00$

0.01

0.24
$0.52 \quad-0.76 \quad 1.28$

$\begin{array}{lll}0.33 & -0.02 & 1.29 \\ 0.10 & -0.14 & 0.26\end{array}$

$\begin{array}{lll}0.21 & -0.41 & 0.41\end{array}$

$\begin{array}{lll}0.29 & -0.62 & 0.53\end{array}$

$0.26 \quad-0.10 \quad 0.90$

$\begin{array}{lll}0.31 & -0.01 & 1.20\end{array}$

$\begin{array}{lll}0.41 & -0.49 & 1.12\end{array}$

$\begin{array}{lll}0.33 & -0.70 & 0.59\end{array}$

$\begin{array}{lll}0.39 & 0.55 & 2.07 \\ 0.23 & 0.85 & 1.77\end{array}$

$\begin{array}{rrr}0.23 & 0.85 & 1.77 \\ 0.07 & -0.09 & 0.19\end{array}$

$\begin{array}{lll}0.53 & -0.96 & 1.11\end{array}$

$\begin{array}{lll}0.18 & -0.40 & 0.29\end{array}$

$\begin{array}{lll}0.29 & -0.37 & 0.78\end{array}$

$\begin{array}{lll}0.14 & 0.09 & 0.65\end{array}$

$\begin{array}{lll}0.22 & -0.03 & 0.83\end{array}$

$\begin{array}{lll}0.24 & 0.21 & 1.14\end{array}$

$\begin{array}{lll}0.22 & -0.36 & 0.52 \\ 0.28 & -0.09 & 0.99\end{array}$

$\begin{array}{lll}0.28 & -0.09 & 0.99 \\ 0.28 & -0.40 & 0.70\end{array}$

$\begin{array}{lll}0.18 & -0.33 & 0.39\end{array}$

$\begin{array}{lll}0.20 & -0.47 & 0.32\end{array}$

$\begin{array}{lll}0.22 & -0.44 & 0.42\end{array}$

$\begin{array}{lll}0.28 & 0.20 & 1.31\end{array}$

$\begin{array}{lll}0.32 & -0.04 & 1.21 \\ 0.21 & -0.54 & 0.30\end{array}$

$\begin{array}{lll}0.21 & -0.54 & 0.30 \\ 0.25 & -0.91 & 0.09\end{array}$

$\begin{array}{lll}0.28 & -0.30 & 0.80\end{array}$

$\begin{array}{lll}0.37 & 0.05 & 1.52\end{array}$

$\begin{array}{lll}0.26 & -0.08 & 0.93\end{array}$

$\begin{array}{lll}0.36 & -0.30 & 1.12\end{array}$

$\begin{array}{lll}0.39 & -1.32 & 0.22\end{array}$

$\begin{array}{lll}0.17 & -0.33 & 0.32\end{array}$

$0.32 \quad-0.61 \quad 0.63$

$\begin{array}{lll}0.06 & 0.13 & 0.36\end{array}$
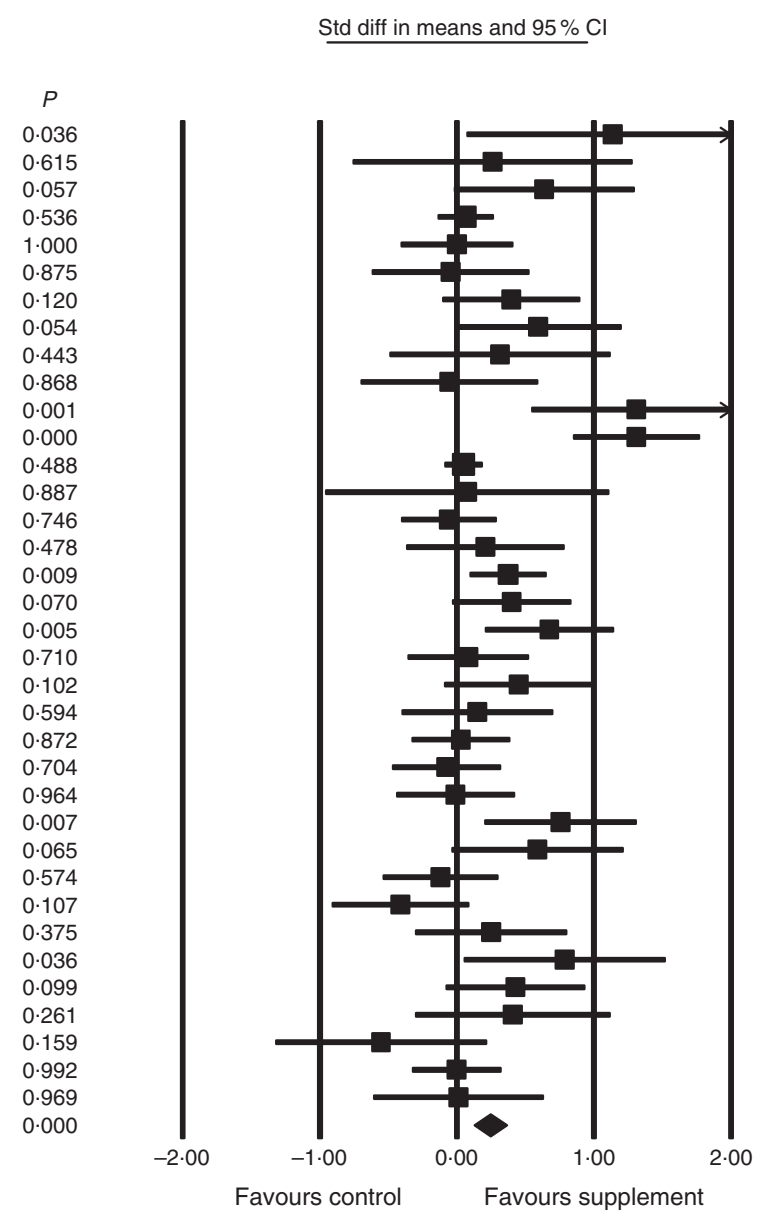

Relative

weight

1.02

1.09

$2 \cdot 10$

5.05

3.47

$2 \cdot 87$

$2 \cdot 32$

$1 \cdot 58$

$2 \cdot 14$

$3 \cdot 11$

$5 \cdot 48$

$1 \cdot 07$

3.91

$2 \cdot 46$

$4 \cdot 44$

3.30

3.06

3.25

$2 \cdot 63$

2.59

3.83
3.56

3.31

2.57

$2 \cdot 22$

3.39

$2 \cdot 87$

2.58

$1 \cdot 80$

$2 \cdot 83$

1.88

$1 \cdot 69$

4.09

$2 \cdot 25$

Fig. 4. Forest plot of effect of supplements on physical function. Random effects model. Heterogeneity: $P 56 \cdot 8, P<0 \cdot 01$.

Group by Supp type

Study name

Amino acid

Amino acid

Amino acid

Amino acid

Amino acid

Amino acid

Amino acid

Amino acid

Amino acid
Abe et al., 2016a

Abe et al., 2016b

Aquiliani et al. 2008

Dal Negro et al., 2012

Dreyer et al., 2013

Kim et al., 2012

Leenders et al., 2011

Rondanelli et al., 2011

Std diff
in means
1.44
0.53
0.67
1.86
0.18
0.72
0.07
1.07
0.82

Statistics for each study

$\begin{array}{crc}\text { SE } & \begin{array}{c}\text { Lower } \\ \text { limit }\end{array} & \begin{array}{c}\text { Upper } \\ \text { limit }\end{array} \\ 0.47 & 0.52 & 2.37 \\ 0.44 & -0.34 & 1.40 \\ 0.34 & 0.02 & 1.33 \\ 0.26 & 1.36 & 2.36 \\ 0.38 & -0.57 & 0.93 \\ 0.24 & 0.25 & 1.19 \\ 0.27 & -0.45 & 0.59 \\ 0.33 & 0.41 & 1.72 \\ 0.24 & 0.35 & 1.28\end{array}$

Std diff in means and $95 \% \mathrm{Cl}$

$P$
0.002
0.234
0.045
0.000
0.632
0.003
0.793
0.001
0.001

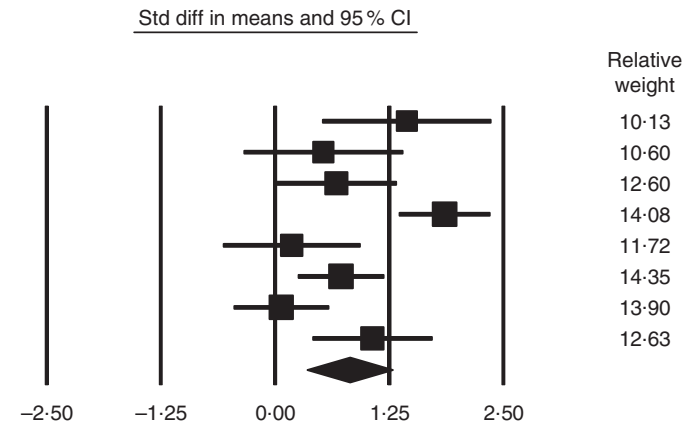

Favours control Favours supplement

Fig. 5. Forest plot of effect of essential amino acids on muscle strength. Random effects model: Heterogeneity: $P 76 \cdot 9, P<0 \cdot 01$.

Other subgroup analysis showed improvements in studies using EAA (SMD $0 \cdot 82 ; 95 \%$ CI $0.35, \quad 1 \cdot 28, \quad P<0 \cdot 01$, significant heterogeneity, $I^{2}=76 \cdot 9, P<0 \cdot 01$ ) (Fig. 5), but not other supplements used. Studies using supplements with or without additional vitamins or minerals were both effective, with greater effect size seen with supplements with no added vitamins and minerals (SMD $0.77 ; 95 \%$ CI $0.22,1 \cdot 33, P<0 \cdot 01$, significant heterogeneity, $\left.I^{2}=82 \cdot 3, P<0 \cdot 01\right)$ than those with added vitamins and minerals (SMD 0.12; $95 \%$ CI 0.00, 0.25, $P=0 \cdot 04$, no evidence of heterogeneity, $I^{2}=27 \cdot 7, P=0 \cdot 11$ ). Supplements were effective in studies with undernourished participants in community (SMD 0.55; 95\% CI 0.09, 1.02, $P=0.02$, significant heterogeneity, $I^{2}=84 \cdot 6, P<0 \cdot 01$ ) or LTC settings (SMD 0.42 ; $95 \%$ CI $0.05,0.79, P=0.03$, significant heterogeneity, $I^{2}=55.7$, $P=0.04)$; in patients not participating in rehabilitation programs (SMD 0.37; 95\% CI 0.13, 0.61, P<0.01, significant heterogeneity, $\left.I^{2}=75 \cdot 8, P<0 \cdot 01\right)$; and in studies measuring upper body muscle strength (online Supplementary Table S3). Metaregression of the protein (slope -0.001 , SE $0.005, P=0.78$ ) or EAA (slope 0.006 , sE $0.071, P=0.94$ ) dose provided in 
Effect of protein and amino acid supplements

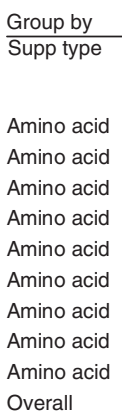

Study name

$\begin{array}{lc} & \begin{array}{c}\text { Std diff } \\ \text { in means }\end{array} \\ \text { Abe et al., 2016a } & 1.14 \\ \text { Abe et al., 2016b } & 0.26 \\ \text { Aquiliani et al., 2008 } & 0.64 \\ \text { Dal Negro et al., 2010 } & 1.31 \\ \text { Dal Negro et al., 2012 } & 1.31 \\ \text { Dreyer et al., 2013 } & 0.07 \\ \text { Kim et al., 2012 } & 0.68 \\ \text { Rondanelli et al., 2011 } & 0.59 \\ & 0.82 \\ & 0.82\end{array}$

Statistics for each study

\begin{tabular}{crr}
\hline SE & $\begin{array}{r}\text { Lower } \\
\text { limit }\end{array}$ & $\begin{array}{r}\text { Uppe } \\
\text { limit }\end{array}$ \\
0.54 & 0.07 & 2.20 \\
0.52 & -0.76 & 1.28 \\
0.33 & -0.02 & 1.29 \\
0.39 & 0.55 & 2.07 \\
0.23 & 0.85 & 1.77 \\
0.53 & -0.96 & 1.11 \\
0.24 & 0.21 & 1.14 \\
0.32 & -0.04 & 1.21 \\
0.15 & 0.52 & 1.12 \\
0.15 & 0.52 & 1.12
\end{tabular}

Std diff in means and $95 \% \mathrm{Cl}$

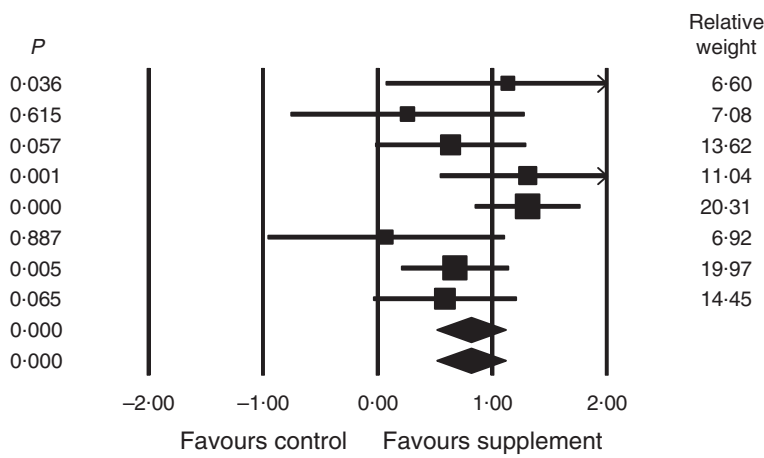

Fig. 6. Forest plot of effect of essential amino acids on physical function. Random effects model: Heterogeneity: $P$ 33.6, $P=0 \cdot 16$.

interventions showed no evidence of dose-response. Duration of intervention also showed no significant effect (slope 0.010, SE $0 \cdot 006, P=0 \cdot 11$ ). Studies ( $n$ ) using a true placebo showed a marginally significant effect on muscle strength (SMD 0.67; $95 \%$ CI $-0 \cdot 08,1 \cdot 41, P=0 \cdot 08$, significant heterogeneity, $I^{2}=88 \cdot 4$, $P<0 \cdot 01)$.

\section{Physical function}

In all, thirty-four studies assessed physical function (analysed $n$ 3396) using a variety of methods. Composite physical performance scores included the physical component sections of the Short Form-12 and -36 Health Surveys, the short physical performance battery, Barthel Index or other measures of ADL. Other tests included walking tests, measured for time, distance or speed; and sitting and standing tests. The effect of supplementation on physical function (Fig. 4) was statistically significant with a small effect size (SMD 0.24; $95 \%$ CI 0.13, 0.36, $P<0 \cdot 01$, significant heterogeneity, $I^{2}=56 \cdot 8, P<0 \cdot 01$ ). Analysis of seven studies with low $\operatorname{ROB}^{(43,46,56,63,64,70,74)}$ indicated no significant effect; however, a small effect was seen in studies with unclear ROB (SMD 0.30; 95\% CI 0.15, 0.45, $P<0.01$, significant heterogeneity, $I^{2}=60 \cdot 7, P<0 \cdot 01$ ). Other subgroup analysis showed improvements in studies using EAA (SMD 0.82; 95\% CI $0.52,1.12, P<0.01$, no evidence of heterogeneity, $I^{2}=33 \cdot 6, P=0 \cdot 16$ ) (Fig. 6) and moderate protein density ONS (SMD $0.15 ; 95 \%$ CI $0.00,0.30, P<0.05$, no evidence of heterogeneity, $I^{2}=14 \cdot 6, P=0 \cdot 31$ ), with marginally significant improvement in higher v. lower protein density ONS (SMD 0.35; $95 \%$ CI $-0.01,0.72, P=0.06$, no evidence of heterogeneity, $I^{2}=11.9, P=0.32$ ), but not other supplement types. Studies using supplements with or without additional vitamins or minerals were both effective, with greater effect seen with supplements with no additional vitamins and minerals (SMD $0 \cdot 83 ; 95 \%$ CI $0.49,1 \cdot 17, P<0 \cdot 01$, no evidence of heterogeneity, $\left.I^{2}=44 \cdot 3, P=0 \cdot 11\right)$ compared with supplements with additional vitamins and minerals (SMD 0.13; 95\% CI 0.04, 0.22, $P<0 \cdot 01$, no evidence of heterogeneity, $I^{2}=24 \cdot 0, P=0 \cdot 12$ ). Supplements were effective in studies undertaken with undernourished participants in community settings (SMD 0.50; 95\% CI 0.17, $0.82, P<0.01$, significant heterogeneity, $\left.I^{2}=76.5, P<0.01\right)$; in patients not participating in rehabilitation programs (SMD 0.31; $95 \%$ CI $0 \cdot 15,0 \cdot 46, P<0 \cdot 01$, significant heterogeneity, $I^{2}=64.9$,
$P<0.01$ ); and in studies measuring physical function through usual physical activity tests, the short physical performance battery, and walking tests (online Supplementary Table S3). Meta-regression of the protein (slope 0.006; SE 0.003, $P>0.05$ ) or EAA (slope -0.039 , sE $0.038, P=0.30$ ) dose provided in interventions showed no evidence of dose-response. Longer intervention length showed marginal effect, with longer study length corresponding to smaller SMD (slope -0.002; SE 0.001, $P=0.02)$. Studies $(n$ 5) using a true placebo showed a significant effect on physical function (SMD 0.67; 95\% CI $0 \cdot 05,1 \cdot 30, P=0 \cdot 04$, significant heterogeneity, $\left.I^{2}=81 \cdot 6, P<0 \cdot 01\right)$.

\section{Discussion}

\section{Overview}

This systematic review and meta-analysis found consistent positive effects, however, significant heterogeneity was evident for the outcomes of muscle strength and physical function, and analysis of only low ROB studies showed no effect on any outcome. The study suggests that supplementation with EAA, and to a lesser extent protein through ONS or protein-rich foods, improves parameters of FFM, muscle strength and physical function for malnourished, frail, sarcopenic, dependent elderly, or those with acute or chronic conditions. This effect was seen even without the addition of RT exercise.

\section{Risk of bias and heterogeneity}

Studies with high ROB were excluded from the analysis ${ }^{(82,83)}$, however, only seven of the thirty-nine studies had low ROB. Inadequate or poor reporting of allocation concealment, outcome data and blinding were common issues found. In many studies, the lack of a placebo with the control group receiving no intervention, or usual care, increases the likelihood of bias towards the intervention group.

For trials with low ROB, there was no significant effect for any outcome measure of FFM (one study, $n$ 65), muscle strength (five studies, $n$ 537) or physical function (seven studies, $n$ 706). Subgroup analysis of trials with unclear ROB showed supplements were effective, with a small effect size similar to the overall result (SMD 0.23-0.31 v. SMD 0.21-0.27 for all analysed). This difference may be due to the smaller number 
of low ROB studies reducing statistical power, compared with the unclear ROB subgroup (fifteen to twenty-seven studies; $n$ 905-2690). In addition, no low ROB studies used EAA, which gave the largest effect in this analysis. The following discussion is based on studies of unclear and low ROB combined.

\section{Most effective supplement}

In predetermined subgroup analysis, we found protein-rich foods improved FFM (SMD 0.26), moderate protein density ONS benefitted physical function (SMD 0.15), but EAA produced the largest change in muscle strength and physical function (SMD 0.82, both).

EAA are required for stimulation of $\mathrm{MPS}^{(12)}$, with approximately $2 \cdot 0-2 \cdot 5 \mathrm{~g}$ leucine proposed to be required ${ }^{(7,84)}$. Five studies using EAA-only formulations, found significant improvements after 6-8 g EAA and $2.5 \mathrm{~g}$ leucine/d in predominantly undernourished patients ${ }^{(14-16,45,78)}$. Leenders et $a{ }^{(80)}$ supplemented leucine only to normally nourished elderly with type 2 diabetes and $1.0 \mathrm{~g}$ protein $/ \mathrm{kg}$ body weight per $\mathrm{d}$, but found no effect of an additional $7.5 \mathrm{~g}$ leucine daily. This may be due to patients normal nutritional status, adequate baseline diets, and the threshold of MPS being reached ${ }^{(85)}$. The result is consistent with recent reviews showing the short term impact of leucine on MPS but not longer term muscle mass or strength effects ${ }^{(86,87)}$

The large effect of EAA, and smaller effect of ONS and proteinrich foods highlight that the effects of protein consumed as whole food and supplements differ. There are many factors influencing MPS, including the quality and source of the protein ingested. These determine the AA composition, leucine concentration, protein digestibility and subsequent availability of absorbed AA for protein synthesis ${ }^{(12,88-93)}$. Jonker et al. recently found that a high-leucine EAA mixture was superior to a similar mixture with the AA composition of complete proteins, in improving wholebody net protein gain ${ }^{(94)}$, and suggests possible benefits and effectiveness of EAA over complete protein ${ }^{(92)}$.

Protein source influences digestion and absorption rates, and the concept of 'fast' and 'slow' proteins ${ }^{(7,95)}$ has emerged. This differentiates proteins based on digestion and absorption kinetics with rapidly digested and absorbed proteins (e.g. whey protein), producing higher post-prandial serum AA concentrations, compared with more slowly digested and absorbed proteins (e.g. casein $)^{(10,89)}$. Research shows higher doses of soya and casein are required to stimulate MPS compared with whey, in part due to faster absorption rates of whey protein ${ }^{(95,96)}$.

Food form also contributes to digestibility, with liquid meal replacements ${ }^{(97)}$ providing greater AA and leucine levels than the same formulation ingested as solid food. Minced meat is also superior to whole steak as it is more rapidly digested, absorbed and provides greater improvement in whole-body protein balance in older men ${ }^{(98)}$.

The interventions using protein-rich foods, which found improvement in FFM, utilised a variety of supplements but many used milk or milk powders, which are high quality proteins containing EAA and leucine with a Digestible Indispensable Amino Acid Score of greater than $1 \cdot 0^{(99)}$. In addition, extra protein was used to fortify usual food, likely at meal times and the dose of protein provided may have reached the threshold for MPS

The small effect seen with ONS compared with EAA interventions may be due to the quality of the protein and the dose administered by ONS. Most ONS products, although easily absorbed liquids, contain mixtures of whole proteins, for example soya and casein, without additional leucine and may have similar digestibility to 'slow' rather than 'fast' proteins. Further, the co-ingestion of macronutrients such as fat may slow gastrointestinal transit.

Three studies investigated whey protein supplements, containing 20-40 g whey protein with no overall effect seen. Bauer et $a l .{ }^{(56)}$ reported significant increase in appendicular FFM and improved grip strength in the whey-supplemented group, and Björkman et al. ${ }^{(57)}$ found significant reduction in ADL assistance needed in the whey protein group, although not the overall ADL score. Only Sugawara et al. ${ }^{(73)}$ found between-group improvements in strength and 6-min walk distance after supplementation with whey containing ONS. Previous studies show approximately $2 \mathrm{~g}$ of leucine, in $20 \mathrm{~g}$ of whey protein, may be required for MPS ${ }^{(84)}$; however, only Bauer et al. ${ }^{(56)}$ provided adequate information on amounts of leucine provided. Further studies are required to ascertain the effect of whey protein supplements, and authors should provide better information of supplement ingredients.

The largest effect of any supplement was seen with EAA for muscle strength and physical function, but not for FFM. Only three studies using EAA assessed FFM, whereas eight studies assessed muscle strength and physical function, and lack of power may have affected results. However, changes in strength and function, without change in FFM have been found previously. It is known that the correlation between muscle mass and strength reduces with age ${ }^{(100)}$, and studies have shown short-term RT increases strength but not muscle size ${ }^{(101)}$. The quality of muscle, including muscle composition, morphology, contractile quality and neural innervation also impact strength $^{(101-103)}$, but muscle mass assessments only determine mass and do not account for these other factors ${ }^{(100)}$. This may contribute to the lack of observed effect of EAA on FFM.

\section{Timing of supplements}

With the exception of one study ${ }^{(50)}$, no studies reported quantity of protein consumed per meal or the effect of protein bolus size, and those reporting timing of intake only reported that consumption was during or between meal times. Bouillanne et $a l .{ }^{(50)}$ investigated 'pulse' ( $72 \%$ of daily protein at midday meal) compared with 'spread' protein (evenly distributed protein intake, with $30 \%$ of daily protein at midday meal), and found improvements in FFM, but not muscle strength or physical function in undernourished hospitalised patients in the pulse group.

\section{Amount of protein required}

Dose-response analysis was conducted and showed no evidence of any effect of daily grams of protein or EAA for any outcome. For EAA, dose varied from 3 to $8 \mathrm{~g}$ in the seven 
studies assessing the outcome of muscle strength, and 3 to $20 \mathrm{~g}$ for the seven studies assessing physical function. The number of studies was small for FFM ( $n$ 3). Patient disease-related factors and nutrient requirements, as well as baseline functional and nutritional status, are likely to be important in determining any effect; however, we were unable to analyse specific doses further, due to the diversity of interventions, patient health conditions and the lack of adequate information provided by studies.

Subgroup analysis of three studies investigated higher $v$. lower protein density intake with different $\mathrm{ONS}^{(59,61,67)}$, and trended towards benefits in physical function from higher energy and protein intake. In these studies, there were differences between the intervention and control group intake, of 8.5-27.6 g protein and 945-1680 kJ (220-400 kcal) energy from supplementation. Marginally beneficial effects on physical function (SMD 0.35, $P=0.06$ ), but not muscle strength were still found, suggesting higher protein and energy, with additional micronutrient intake, remains beneficial for physical function. The inclusion of these three studies in the full analysis may have reduced the overall result found for muscle strength and physical function, as the additional protein and nutrients in the lower protein group may have reduced the differences seen between groups.

\section{Contribution of other nutrients}

Specific vitamin supplementation was uncommon and most studies provided supplements with small additional amounts of a variety of nutrients, supplied by complete ONS or protein-rich foods containing other nutrients, for example addition of milk protein or ricotta cheese also adds energy, $\mathrm{Ca}$ and vitamin $\mathrm{B}_{12}$. Complete ONS contain a complete range of micronutrients, along with energy. Only protein-rich foods, with intrinsic nutrient content, provided a small improvement in FFM; however, no effect was found when only true placebo studies were analysed. For muscle strength and physical function, supplements with and without additional nutrients were both effective. In fact, a greater effect was seen in studies using supplements with no added nutrients, likely due to inclusion of EAA studies in this subgroup, as EAAs independently had the largest effect on muscle strength and physical function. Analysis of studies using a true placebo, found a marginal beneficial effect for muscle strength and a large positive effect for physical function (SMD 0.67, $P=0.08$ and SMD $0.67, P=0.04$ ), respectively. Four of the five true placebo studies for these analyses used EAA and support the conclusion that EAA rather than other nutrients were the effective component.

There is evidence that vitamin D impacts skeletal muscle. A recent meta-analysis ${ }^{(104)}$ found a small positive effect of vitamin D supplementation on global muscle strength (SMD $0 \cdot 17$ ) with greater benefit in elders with 25 -hydroxyvitamin D level $<30 \mathrm{nmol} / 1$. In our meta-analysis, four studies supplemented with vitamin $\mathrm{D}$ additional to that intrinsically provided by the supplement, but were not designed to determine effect of vitamin $\mathrm{D}^{(56,70,71,79)}$ and we were unable to quantify any effect. Only one study, supplementing with EAA and vitamin $\mathrm{D}^{(79)}$ found an improvement in hand grip strength and walk time compared with the no treatment control group.
Energy intake is also important to consider. The provision of additional energy as well as protein was common, particularly if ONS, milk-based drinks or food fortification was used. Protein and EAA are required for cellular functions as well as formation of muscle. However, in times of energy deficit, protein is utilised as an energy source and muscle breakdown occurs ${ }^{(105)}$. Thus, an energy deficit accelerates muscle protein breakdown and inhibits MPS, which may partly explain why undernourished subjects, most likely in energy and protein deficit, were the most to benefit from supplementation. Additional energy from EAA supplements is unlikely to impact energy intake as the energy provided is only $184-355 \mathrm{~kJ}(44-85 \mathrm{kcal}) / \mathrm{d}$. In contrast, ONS provided between 623-5040 kJ (149-1200 kcal)/d, but was less effective than EAA in producing an effect on muscle strength and function.

\section{Duration of intervention}

Duration of intervention was investigated and a marginal negative effect of longer duration was found for physical function, but no effect was seen for other outcome measures. This suggests intervention time had little or no impact on shortterm outcomes, with no blunting of effect due to duration of supplementation. The use of ONS for 6 weeks and 12 months is acceptable to patients and effective in improving both nutritional status and reducing rates of hospital readmissions ${ }^{(19,106)}$. The time period for improvements in muscle function may be weeks, but continued improvement is unlikely once the individual's threshold of strength and function has been reached. However, additional nutrients may be beneficial to support continued muscle strength and function in the longer term, unless other effective strategies, such as RT are incorporated.

\section{Participants experiencing the greatest benefits}

It is important to identify patient groups where interventions are most efficacious. Subgroup analysis of studies in undernourished, but not nourished community-dwelling elderly indicated significant benefits on muscle strength (SMD 0.55) and physical function (SMD 0.50). Muscle strength was also improved in undernourished elderly in LTC (SMD 0.42), indicating that undernourished groups may experience the greatest benefits from supplementation.

Undernourished individuals generally have lower dietary intakes and higher requirements, often due to disease, for example wound/fracture healing, catabolic effects of inflammatory disease or for whole body protein repletion ${ }^{(7,10)}$. In situations of energy deficit, muscle protein breakdown increases $^{(105)}$ and more protein is needed to compensate for the use of protein as an energy source. Our result is consistent with a Cochrane review finding that undernourished patients benefitted most from supplementation ${ }^{(47)}$. Nutritional status was defined either by the individual trial authors or by BMI measures, and this is not ideal. Future studies should use current consensus definitions or standard tools to better classify nutritional status, frailty and/or sarcopenia. Few studies reported background dietary intake and this is an important modulator and should be provided in future studies. 


\section{Rehabilitation combined with nutrition supplementation}

Supplementation with EAA and protein without rehabilitation exercise produced a small effect (SMD 0.23-0.37). We found effect sizes for the outcomes of muscle strength and physical function, but not FFM, were significant in studies without rehabilitation, but not in studies including rehabilitation. Supplements have been shown to augment rehabilitation effects on muscle strength and physical function ${ }^{(73,107)}$, and the lack of effect may be due to the smaller number of studies within the rehabilitation subgroup ( $n$ 3-12; studies without rehabilitation $n$ 13-22).

\section{Concerns related to higher protein intake}

The thirty-nine studies included elderly from age 60 to 103 years, and other than mild gastrointestinal upset, no complications were reported. The most common concern related to higher protein intake is kidney function deterioration and this topic has been addressed in recent reviews ${ }^{(7,108)}$. These suggest $1 \cdot 0-1 \cdot 2 \mathrm{~g}$ protein $/ \mathrm{kg}$ body weight per $\mathrm{d}$ is suitable for elderly people without a negative impact on kidney function being seen. The International Society of Renal Nutrition and Metabolism $^{(109)}$ recently published guidelines for chronic kidney disease patients, and recommend $1.0 \mathrm{~g}$ protein $/ \mathrm{kg}$ body weight per $\mathrm{d}$ for patients with coexisting disease or injury. Given this, additional protein is unlikely to have negative consequences, particularly in undernourished patients. The use of EAA supplements, effective in smaller doses than whole proteincontaining foods or ONS, may be a more suitable yet effective alternative to higher protein diets.

\section{Study weights}

Individual study weights may have impacted the overall effect size; however, sensitivity analysis that removed individual studies for each outcome showed only a small difference in SMD and marginal change in significance levels, with all SMDs remaining significant. One study, Dal Negro et $a l .{ }^{(78)}$, with a mid-range weighting of 3.69 for muscle strength, produced the highest individual SMD; when this study was removed during sensitivity analysis, the overall SMD was reduced from $0.27(P<0.01)$ to $0.19(P<0.01)$. For physical function, removal of both Dal Negro et $a l^{(16)}$ (weighting of 1.7) and Dal Negro et $a l^{(78)}$ (weighting of $3 \cdot 11$ ), reduced overall SMD from $0.24(P<0.01)$ to $0.17(P<0 \cdot 01)$. Both studies supplemented EAA to sarcopenic patients with chronic obstructive pulmonary disease.

\section{Limitations}

As previously discussed, this meta-analysis is limited by the varied study quality with only seven low ROB studies, which limits the strength of the conclusions, and may lead to overestimation of intervention effects. This should be considered in the interpretation of results. Only trials published in English were included and the search strategy used a limit of 65 years and older, which may have eliminated some studies. However, all included papers and reviews found were hand-searched for additional studies. Heterogeneity was significant for analyses of muscle strength and physical function due to wide variation in outcome measurements, participant characteristics and intervention supplements. Thus, a random effects model was used for all statistical analyses, and pre-determined subgroup analyses according to setting, nutritional status, intervention type and duration of intervention were conducted. Additional post-hoc analysis to determine the effect of true placebo studies, additional nutrients, and dose-response analysis were undertaken.

\section{Conclusions}

Given the ROB of the studies, no definitive conclusions can be made regarding the effects of specific supplements. This meta-analysis does however, provide preliminary data suggesting that EAA may be the most effective supplements and undernourished subjects are the most likely to benefit. More studies with low ROB, using a placebo control group, and adequate reporting of intervention characteristics and dietary intakes are needed. Appropriate interventions in this highly vulnerable group of participants will assist in the maintenance or improvement of strength and functional status, and is important from both a clinical and public health perspective.

\section{Acknowledgements}

The authors would like to thank the University of Sydney Medical Librarians for their assistance with database searching.

There were no funding or grants for the completion of this study.

F. O. L. and B. D. designed the study; H. C., J. K., C. U., P. P. and F. O. L. performed the data extraction; P. P., F. O. L., V. H. and H. C. analysed the data; H. C., F. O. L. and V. H. drafted the manuscript and all authors read and approved the final manuscript.

The authors declare that there are no conflicts of interest.

\section{Supplementary material}

For supplementary material/s referred to in this article, please visit https://doi.org/10.1017/S0007114517003816

\section{References}

1. Cruz-Jentoft AJ, Baeyens JP, Bauer JM, et al. (2010) Sarcopenia: European consensus on definition and diagnosis: Report of the European Working Group on Sarcopenia in Older People. Age Ageing 39, 412-423.

2. Cruz-Jentoft AJ, Landi F, Topinkova E, et al. (2010) Understanding sarcopenia as a geriatric syndrome. Curr Opin Clin Nutr Metab Care 13, 1-7.

3. Janssen I, Shepard DS, Katzmarzyk PT, et al. (2004) The healthcare costs of sarcopenia in the United States. $J \mathrm{Am}$ Geriatr Soc 52, 80-85.

4. Morley JE, Anker SD \& von Haehling S (2014) Prevalence, incidence, and clinical impact of sarcopenia: facts, numbers, and epidemiology-update 2014. J Cachexia Sarcopenia Muscle 5, 253-259.

5. National Institute on Aging, National Institutes of Health \& US Department of Health and Human Services et al. (2011) 
Humanity's Aging. Global Health and Aging. https://www. nia.nih.gov/research/publication/global-health-and-aging/ humanitys-aging (accessed December 2015).

6. Paddon-Jones D \& van Loon L (2012) Nutritional approaches to treating sarcopenia. In Sarcopenia, pp. 275-295 [AJ CruzJentoft and JE Morley, editors]. New York: John Wiley \& Sons, Ltd.

7. Bauer J, Biolo G, Cederholm T, et al. (2013) Evidence-based recommendations for optimal dietary protein intake in older people: a position paper from the prot-age study group. J Am Med Dir Assoc 14, 542-559.

8. Fujita S \& Volpi E (2004) Nutrition and sarcopenia of ageing. Nutr Res Rev 17, 69-76.

9. Paddon-Jones D \& Rasmussen BB (2009) Dietary protein recommendations and the prevention of sarcopenia. Curr Opin Clin Nutr Metab Care 12, 86-90.

10. Nowson C \& O'Connell S (2015) Protein requirements and recommendations for older people: a review. Nutrients $\mathbf{7}$, 6874-6899.

11. Rolland Y, Dupuy C, Abellan van Kan G, et al. (2011) Treatment strategies for sarcopenia and frailty. Med Clin North Am 95, 427-438.

12. Volpi E, Kobayashi H, Sheffield-Moore M, et al. (2003) Essential amino acids are primarily responsible for the amino acid stimulation of muscle protein anabolism in healthy elderly adults. Am J Clin Nutr 78, 250-258.

13. Katsanos CS, Kobayashi H, Sheffield-Moore M, et al. (2006) A high proportion of leucine is required for optimal stimulation of the rate of muscle protein synthesis by essential amino acids in the elderly. Am J Physiol Endocrinol Metab 291, E381-E387.

14. Aquilani R, Opasich C, Gualco A, et al. (2008) Adequate energy-protein intake is not enough to improve nutritional and metabolic status in muscle-depleted patients with chronic heart failure. Eur J Heart Fail 10, $1127-1135$.

15. Rondanelli M, Opizzi A, Antoniello N, et al. (2011) Effect of essential amino acid supplementation on quality of life, amino acid profile and strength in institutionalized elderly patients. Clin Nutr 30, 571-577.

16. Dal Negro RW, Aquilani R, Bertacco S, et al. (2010) Comprehensive effects of supplemented essential amino acids in patients with severe COPD and sarcopenia. Monaldi Arch Chest Dis 73, 25-33.

17. Symons TB, Sheffield-Moore M, Wolfe RR, et al. (2009) A moderate serving of high-quality protein maximally stimulates skeletal muscle protein synthesis in young and elderly subjects. J Am Diet Assoc 109, 1582-1586.

18. Bouillanne O, Neveux N, Nicolis I, et al. (2014) Long-lasting improved amino acid bioavailability associated with protein pulse feeding in hospitalized elderly patients: a randomized controlled trial. Nutrition 30, 544-550.

19. Cawood AL, Elia M \& Stratton RJ (2012) Systematic review and meta-analysis of the effects of high protein oral nutritional supplements. Ageing Res Rev 11, 278-296.

20. Malafarina V, Uriz-Otano F, Iniesta R, et al. (2013) Effectiveness of nutritional supplementation on muscle mass in treatment of sarcopenia in old age: a systematic review. J Am Med Dir Assoc 14, 10-17.

21. Cermak NM, Res PT, De Groot LCPGM, et al. (2012) Protein supplementation augments the adaptive response of skeletal muscle to resistance-type exercise training: a meta-analysis. Am J Clin Nutr 96, 1454-1464.

22. Finger D, Goltz F, Umpierre D, et al. (2015) Effects of protein supplementation in older adults undergoing resistance training: a systematic review and meta-analysis. Sports Med 45, 245-255.
23. Moher D, Liberati A, Tetzlaff J, et al. (2009) Preferred reporting items for systematic reviews and meta-analyses: the PRISMA statement. Ann Intern Med 151, 264-269, w264.

24. Rhea MR, Alvar BA, Burkett LN, et al. (2003) A meta-analysis to determine the dose response for strength development. Med Sci Sports Exerc 35, 456-464.

25. The Cochrane Collaboration (2011) The Cochrane Collaboration's tool for assessing risk of bias. In Cochrane Handbook for Systematic Reviews of Interventions, chapter 5, section 8. http://handbook-5-1.cochrane.org/chapter_8/ 8_assessing_risk_of_bias_in_included_studies.htm

26. Baldi S, Aquilani R, Pinna GD, et al. (2010) Fat-free mass change after nutritional rehabilitation in weight losing COPD: role of insulin, C-reactive protein and tissue hypoxia. Int J Chron Obstruct Pulmon Dis 5, 29-39.

27. Feldblum I, German L, Castel H, et al. (2011) Individualized nutritional intervention during and after hospitalization: the nutrition intervention study clinical trial. J Am Geriatr Soc 59, $10-17$.

28. Gray-Donald K, Payette H \& Boutier V (1995) Randomized clinical trial of nutritional supplementation shows little effect on functional status among free-living frail elderly. $J$ Nutr 125, 2965-2971.

29. Hampson G, Martin FC, Moffat K, et al. (2003) Effects of dietary improvement on bone metabolism in elderly underweight women with osteoporosis: a randomised controlled trial. Osteoporos Int 14, 750-756.

30. Manders M, De Groot LC, Hoefnagels WH, et al. (2009) The effect of a nutrient dense drink on mental and physical function in institutionalized elderly people. J Nutr Health Aging 13, 760-767.

31. Neelemaat F, Bosmans JE, Thijs A, et al. (2011) Postdischarge nutritional support in malnourished elderly individuals improves functional limitations. J Am Med Dir Assoc 12, 295-301.

32. Price R, Daly F, Pennington CR, et al. (2005) Nutritional supplementation of very old people at hospital discharge increases muscle strength: a randomised controlled trial. Gerontology 51, 179-185.

33. Stange I, Bartram M, Liao Y, et al. (2013) Effects of a lowvolume, nutrient- and energy-dense oral nutritional supplement on nutritional and functional status: a randomized, controlled trial in nursing home residents. J Am Med Dir Assoc 14, 628.e621-628.

34. Stow R, Ives N, Smith C, et al. (2015) A cluster randomised feasibility trial evaluating nutritional interventions in the treatment of malnutrition in care home adult residents. Trials 16, 433 .

35. Tidermark J, Ponzer S, Carlsson P, et al. (2004) Effects of protein-rich supplementation and nandrolone in lean elderly women with femoral neck fractures. Clin Nutr 23, 587-596.

36. Woo J, Ho SC, Mak YT, et al. (1994) Nutritional status of elderly patients during recovery from chest infection and the role of nutritional supplementation assessed by a prospective randomized single-blind trial. Age Ageing 23, 40-48.

37. Wouters-Wesseling W, Slump E, Kleijer CN, et al. (2006) Early nutritional supplementation immediately after diagnosis of infectious disease improves body weight in psychogeriatric nursing home residents. Aging Clin Exp Res 18, $70-74$.

38. Wouters-Wesseling W, Van Hooijdonk C, Wagenaar L, et al. (2003) The effect of a liquid nutrition supplement on body composition and physical functioning in elderly people. Clin Nutr 22, 371-377.

39. Wouters-Wesseling W, Wouters AE, Kleijer CN, et al. (2002) Study of the effect of a liquid nutrition supplement on the 
nutritional status of psycho-geriatric nursing home patients. Eur J Clin Nutr 56, 245-251.

40. Boreinstein M, Hedges LV, Higgins JPT, et al. (2009) Intro duction to Meta-Analysis. Chichester: John Wiley and Sons, Ltd.

41. Kim CO \& Lee KR (2013) Preventive effect of protein-energy supplementation on the functional decline of frail older adults with low socioeconomic status: a community-based randomized controlled study. J Gerontol A Biol Sci Med Sci 68, 309-316.

42. Tieland M, Borgonjen-Van Den Berg KJ, Van Loon LJC, et al. (2012) Dietary protein intake in community-dwelling, frail, and institutionalized elderly people: scope for improvement. Eur J Nutr 51, 173-179.

43. Zak M, Swine C \& Grodzicki T (2009) Combined effects of functionally-oriented exercise regimens and nutritional supplementation on both the institutionalised and free-living frail elderly (double-blind, randomised clinical trial). $B M C$ Public Health 9, 39.

44. Fiatarone MA, O'Neill EF, Ryan ND, et al. (1994) Exercise training and nutritional supplementation for physical frailty in very elderly people. $N$ Engl J Med 330, 1769-1775.

45. Kim HK, Suzuki T, Saito K, et al. (2012) Effects of exercise and amino acid supplementation on body composition and physical function in community-dwelling elderly Japanese sarcopenic women: a randomized controlled trial. J Am Geriatr Soc 60, 16-23.

46. Rosendahl E, Lindelof N, Littbrand H, et al. (2006) Highintensity functional exercise program and protein-enriched energy supplement for older persons dependent in activities of daily living: a randomised controlled trial. Aust J Physiother 52, 105-113.

47. Milne AC, Potter J, Vivanti A, et al. (2009) Protein and energy supplementation in elderly people at risk from malnutrition. Cochrane Database Syst Rev, issue 2, CD003288.

48. Deeks JJ, Higgins JPT \& Altman DG (2011) Analysing data and undertaking meta-analyses. In Cochrane Handbook for Systematic Reviews of Interventions, 5.0.1 [JJ Deeks and JPT Higgins, editors]. The Cochrane Collaboration.

49. Durlak JA (2009) How to select, calculate, and interpret effect sizes. J Pediatr Psychol 34, 917-928.

50. Bouillanne O, Curis E, Hamon-Vilcot B, et al. (2013) Impact of protein pulse feeding on lean mass in malnourished and at-risk hospitalized elderly patients: a randomized controlled trial. Clin Nutr 32, 186-192.

51. Payette H, Boutier V, Coulombe C, et al. (2002) Benefits of nutritional supplementation in free-living, frail, undernourished elderly people: a prospective randomized community trial. J Am Diet Assoc 102, 1088-1095.

52. Dreyer HC, Strycker LA, Senesac HA, et al. (2013) Essential amino acid supplementation in patients following total knee arthroplasty. J Clin Invest 123, 4654-4666.

53. Smoliner C, Norman K, Scheufele R, et al. (2008) Effects of food fortification on nutritional and functional status in frail elderly nursing home residents at risk of malnutrition. Nutrition 24, 1139-1144.

54. Aleman-Mateo H, Macias L, Esparza-Romero J, et al. (2012) Physiological effects beyond the significant gain in muscle mass in sarcopenic elderly men: evidence from a randomized clinical trial using a protein-rich food. Clin Interv Aging 7, 225-234.

55. Bakhtiari A, Yassin Z, Hanachi P, et al. (2012) Effects of soy on body composition: a 12 -week randomized controlled trial among Iranian elderly women with metabolic syndrome. Iran J Public Health 41, 9-18.

56. Bauer JM, Verlaan S, Bautmans I, et al. (2015) Effects of a vitamin $d$ and leucine-enriched whey protein nutritional supplement on measures of sarcopenia in older adults, the provide study: a randomized, double-blind, placebocontrolled trial. J Am Med Dir Assoc 16, 740-747.

57. Björkman MP, Finne-Soveri H \& Tilvis RS (2012) Whey protein supplementation in nursing home residents. A randomized controlled trial. Eur Geriatr Med 3, 161-166.

58. Bonnefoy M, Cornu C, Normand S, et al. (2003) The effects of exercise and protein-energy supplements on body composition and muscle function in frail elderly individuals: a long-term controlled randomised study. Br J Nutr 89, 731-739.

59. Cameron ID, Kurrle SE, Uy C, et al. (2011) Effectiveness of oral nutritional supplementation for older women after a fracture: rationale, design and study of the feasibility of a randomized controlled study. BMC Geriatr 11, 32.

60. Chapman IM, Visvanathan R, Hammond AJ, et al. (2009) Effect of testosterone and a nutritional supplement, alone and in combination, on hospital admissions in undernourished older men and women. Am J Clin Nutr 89, 880-889.

61. Collins CE, Kershaw J \& Brockington S (2005) Effect of nutritional supplements on wound healing in home-nursed elderly: a randomized trial. Nutrition 21, 147-155.

62. Dangour AD, Albala C, Allen E, et al. (2011) Effect of a nutrition supplement and physical activity program on pneumonia and walking capacity in Chilean older people: a factorial cluster randomized trial. PLoS Med 8, e1001023.

63. Espaulella J, Guyer H, Diaz-Escriu F, et al. (2000) Nutritional supplementation of elderly hip fracture patients. A randomized, double-blind, placebo-controlled trial. Age Ageing 29, 425-431.

64. Gariballa S \& Forster S (2007) Effects of dietary supplements on depressive symptoms in older patients: a randomised double-blind placebo-controlled trial. Clin Nutr 26, 545-551.

65. Lauque S, Arnaud-Battandier F, Gillette S, et al. (2004) Improvement of weight and fat-free mass with oral nutritional supplementation in patients with Alzheimer's disease at risk of malnutrition: a prospective randomized study. J Am Geriatr Soc 52, 1702-1707.

66. Lauque S, Arnaud-Battandier F, Mansourian R, et al. (2000) Protein-energy oral supplementation in malnourished nursing-home residents. A controlled trial. Age Ageing 29, 51-56.

67. McMurdo ME, Price RJ, Shields M, et al. (2009) Should oral nutritional supplementation be given to undernourished older people upon hospital discharge? A controlled trial. J Am Geriatr Soc 57, 2239-2245.

68. Miller MD, Crotty M, Whitehead C, et al. (2006) Nutritional supplementation and resistance training in nutritionally at risk older adults following lower limb fracture: a randomized controlled trial. Clin Rehabil 20, 311-323.

69. Myint MWW, Wu J, Wong E, et al. (2013) Clinical benefits of oral nutritional supplementation for elderly hip fracture patients: a single blind randomised controlled trial. Age Ageing 42, 39-45.

70. Ng TP, Feng L, Nyunt MSZ, et al. (2015) Nutritional, physical, cognitive, and combination interventions and frailty reversal among older adults: a randomized controlled trial. Am J Med 128, 1225-1236.

71. Persson M, Hytter-Landahl A, Brismar K, et al. (2007) Nutritional supplementation and dietary advice in geriatric patients at risk of malnutrition. Clin Nutr 26, 216-224.

72. Schurch MA, Rizzoli R, Slosman D, et al. (1998) Protein supplements increase serum insulin-like growth factor-I levels and attenuate proximal femur bone loss in patients 
with recent hip fracture. A randomized, double-blind, placebo-controlled trial. Ann Intern Med 128, 801-809.

73. Sugawara K, Takahashi H, Kashiwagura T, et al. (2012) Effect of anti-inflammatory supplementation with whey peptide and exercise therapy in patients with COPD. Respir Med 106, 1526-1534.

74. Tieland M, van de Rest O, Dirks ML, et al. (2012) Protein supplementation improves physical performance in frail elderly people: a randomized, double-blind, placebocontrolled trial. J Am Med Dir Assoc 13, 720-726.

75. Volkert D, Hubsch S, Oster P, et al. (1996) Nutritional support and functional status in undernourished geriatric patients during hospitalization and 6-month follow-up. Aging (Milano) 8, 386-395.

76. Yamada M, Nishiguchi S, Fukutani N, et al. (2015) Mailbased intervention for sarcopenia prevention increased anabolic hormone and skeletal muscle mass in communitydwelling Japanese older adults: The INE (Intervention by Nutrition and Exercise) Study. J Am Med Dir Assoc 16, 654-660

77. Bonnefoy M, Laville M, Ecochard R, et al. (2010) Effects of branched amino acids supplementation in malnourished elderly with catabolic status. J Nutr Health Aging $\mathbf{1 4}$ 579-584.

78. Dal Negro RW, Testa A, Aquilani R, et al. (2012) Essential amino acid supplementation in patients with severe COPD: a step towards home rehabilitation. Monaldi Arch Chest Dis 77, 67-75

79. Abe S, Ezaki O \& Suzuki M (2016) Medium-chain triglycerides in combination with leucine and vitamin d increase muscle strength and function in frail elderly adults in a randomized controlled trial. J Nutr 146, 1017-1026.

80. Leenders M, Verdijk LB, van der Hoeven L, et al. (2011) Prolonged leucine supplementation does not augment muscle mass or affect glycemic control in elderly type 2 diabetic men. J Nutr 141, 1070-1076.

81. Gariballa S \& Forster S (2007) Dietary supplementation and quality of life of older patients: a randomized, doubleblind, placebo-controlled trial. I Am Geriatr Soc $\mathbf{5 5}$, 2030-2034.

82. The Cochrane Collaboration (2011) Summary assessments of risk of bias. In Cochrane Handbook for Systematic Reviews of Interventions, 5.1.0 ed. [JPT Higgins and S Green, editors]. The Cochrane Collaboration. http://training.cochrane.org/ handbook

83. Higgins JPT (2012) Incorporating 'risk of bias' assessments into meta-analyses. http://methods.cochrane.org/sites/methods. cochrane.org.bias/files/public/uploads/JH\%20incorporating\% 20in\%20meta-analyses.pdf (accessed November 2016).

84. Breen L \& Phillips SM (2011) Skeletal muscle protein metabolism in the elderly: Interventions to counteract the 'anabolic resistance' of ageing. Nutr Metab 8, 68.

85. Paddon-Jones D, Campbell WW, Jacques $\mathrm{PF}$, et al. (2015) Protein and healthy aging. Am J Clin Nutr 101, 1339S-1345S.

86. Xu ZR, Tan ZJ, Zhang Q, et al. (2015) The effectiveness of leucine on muscle protein synthesis, lean body mass and leg lean mass accretion in older people: a systematic review and meta-analysis. Br J Nutr 113, 25-34.

87. Ham DJ, Caldow MK, Lynch GS, et al. (2014) Leucine as a treatment for muscle wasting: a critical review. Clin Nutr 33, 937-945.

88. Deutz NE, Bauer JM, Barazzoni R, et al. (2014) Protein intake and exercise for optimal muscle function with aging: recommendations from the ESPEN Expert Group. Clin Nutr 33, 929-936.
89. Walrand S, Gryson C, Salles J, et al. (2016) Fast-digestive protein supplement for ten days overcomes muscle anabolic resistance in healthy elderly men. Clin Nutr $\mathbf{3 5}$, 660-668.

90. Phillips SM, Chevalier S \& Leidy HJ (2016) Protein 'requirements' beyond the RDA: implications for optimizing health. Appl Physiol Nutr Metab 41, 565-572.

91. Phillips SM, Tang JE \& Moore DR (2009) The role of milkand soy-based protein in support of muscle protein synthesis and muscle protein accretion in young and elderly persons. J Am Coll Nutr 28, 343-354.

92. Paddon-Jones D, Sheffield-Moore M, Katsanos CS, et al. (2006) Differential stimulation of muscle protein synthesis in elderly humans following isocaloric ingestion of amino acids or whey protein. Exp Gerontol 41, 215-219.

93. Tang JE \& Phillips SM (2009) Maximizing muscle protein anabolism: the role of protein quality. Curr Opin Clin Nutr Metab Care 12, 66-71.

94. Jonker R, Deutz NE, Erbland ML, et al. (2017) Effectiveness of essential amino acid supplementation in stimulating whole body net protein anabolism is comparable between COPD patients and healthy older adults. Metabolism 69, 120-129.

95. Boirie Y, Dangin M, Gachon P, et al. (1997) Slow and fast dietary proteins differently modulate postprandial protein accretion. Proc Natl Acad Sci 94, 14930-14935.

96. Witard OC, Wardle SL, Macnaughton LS, et al. (2016) Protein considerations for optimising skeletal muscle mass in healthy young and older adults. Nutrients $\mathbf{8}, 181$.

97. Conley TB, Apolzan JW, Leidy HJ, et al. (2011) Effect of food form on postprandial plasma amino acid concentrations in older adults. Br J Nutr 106, 203-207.

98. Pennings B, Groen BB, van Dijk JW, et al. (2013) Minced beef is more rapidly digested and absorbed than beef steak, resulting in greater postprandial protein retention in older men. Am J Clin Nutr 98, 121-128.

99. Phillips SM (2017) Current concepts and unresolved questions in dietary protein requirements and supplements in adults. Front Nutr $\mathbf{4}, 13$.

100. Correa-de-Araujo R, Harris-Love MO, Miljkovic I, et al. (2017) The need for standardized assessment of muscle quality in skeletal muscle function deficit and other aging-related muscle dysfunctions: a symposium report. Front Physiol $\mathbf{8}, 87$.

101. Beyer KS, Fukuda DH, Boone CH, et al. (2016) Short-term unilateral resistance training results in cross education of strength without changes in muscle size, activation, or endocrine response. J Strength Cond Res 30, 1213-1223.

102. Reid KF \& Fielding RA (2012) Skeletal muscle power: a critical determinant of physical functioning in older adults. Exerc Sport Sci Rev 40, 4-12.

103. Clark BC \& Manini TM (2010) Functional consequences of sarcopenia and dynapenia in the elderly. Curr Opin Clin Nutr Metab Care 13, 271-276.

104. Beaudart C, Buckinx F, Rabenda V, et al. (2014) The effects of vitamin $\mathrm{D}$ on skeletal muscle strength, muscle mass, and muscle power: a systematic review and meta-analysis of randomized controlled trials. J Clin Endocrinol Metab 99, 4336-4345.

105. Murphy CH, Churchward-Venne TA, Mitchell CJ, et al. (2015) Hypoenergetic diet-induced reductions in myofibrillar protein synthesis are restored with resistance training and balanced daily protein ingestion in older men. Am J Physiol Endocrinol Metab 308, E734-e743.

106. Stratton RJ, Hebuterne X \& Elia M (2013) A systematic review and meta-analysis of the impact of oral nutritional 
supplements on hospital readmissions. Ageing Res Rev $\mathbf{1 2}$ 884-897.

107. Creutzberg EC, Wouters EFM, Mostert R, et al. (2003) Efficacy of nutritional supplementation therapy in depleted patients with chronic obstructive pulmonary disease. Nutrition 19, 120-127.

108. Gaffney-Stomberg E, Insogna KL, Rodriguez NR, et al. (2009) Increasing dietary protein requirements in elderly people for optimal muscle and bone health. I Am Geriatr Soc 57, 1073-1079.

109. Ikizler TA, Cano NJ, Franch H, et al. (2013) Prevention and treatment of protein energy wasting in chronic kidney disease patients: a consensus statement by the International Society of Renal Nutrition and Metabolism. Kidney Int 84, 1096-1107. 